\title{
KINETIC STRUCTURE SIMULATIONS OF NEMATIC POLYMERS IN PLANE COUETTE CELLS. II: IN-PLANE STRUCTURE TRANSITIONS*
}

\author{
M. GREGORY FOREST ${ }^{\dagger}$, RUHAI ZHOU ${ }^{\ddagger}$, AND QI WANG ${ }^{\S}$
}

\begin{abstract}
Nematic, or liquid crystalline, polymer (LCP) composites are composed of large aspect ratio rod-like or platelet macromolecules. This class of nanocomposites exhibits tremendous potential for high performance material applications, ranging across mechanical, electrical, piezoelectric, thermal, and barrier properties. Fibers made from nematic polymers have set synthetic materials performance standards for decades. The current target is to engineer multifunctional films and molded parts, for which processing flows are shear-dominated. Nematic polymer films inherit anisotropy from collective orientational distributions of the molecular constituents and develop heterogeneity on length scales that are, as yet, not well understood and thereby uncontrollable. Rigid LCPs in viscous solvents have a theoretical and computational foundation from which one can model parallel plate Couette cell experiments and explore anisotropic structure generation arising from nonequilibrium interactions between hydrodynamics, molecular short- and long-range elasticity, and confinement effects. Recent progress on the longwave limit of homogeneous nematic polymers in imposed simple shear and linear planar flows [R. G. Larson and H. Ottinger, Macromolecules, 24 (1991), pp. 6270-6282], [V. Faraoni, M. Grosso, S. Crescitelli, and P. L. Maffettone, J. Rheol., 43 (1999), pp. 829-843], [M. Grosso, R. Keunings, S. Crescitelli, and P. L. Maffettone, Phys. Rev. Lett., 86 (2001), pp. 3184-3187], [M. G. Forest, Q. Wang, and R. Zhou, Rheol. Acta, 43 (2004), pp. 17-37], [M. G. Forest, Q. Wang, and R. Zhou, Rheol. Acta, 44 (2004), pp. 80-93], [M. G. Forest, Q. Wang, R. Zhou, and E. Choate, J. Non-Newtonian Fluid Mech., 118 (2004), pp. 17-31], [M. G. Forest, R. Zhou, and Q. Wang, Phys. Rev. Lett., 93 (2004), 088301] provides resolved kinetic simulations of the molecular orientational distribution. These results characterize anisotropy and dynamic attractors of sheared bulk domains and underscore limitations of mesoscopic models for orientation of the rigid rod or platelet ensembles. In this paper, we apply our resolved kinetic structure code [R. Zhou, M. G. Forest, and Q. Wang, Multiscale Model. Simul., 3 (2005), pp. 853-870] to model onset and saturation of heterogeneity in the orientational distribution by coupling a distortional elasticity potential (with distinct elasticity constants) and anchoring conditions in a plane Couette cell. For this initial study, the flow field is imposed and the orientational distribution is confined to the shear deformation plane, which affords comparison with seminal [T. Tsuji and A. D. Rey, Phys. Rev. E (3), 62 (2000), pp. 8141-8151] as well as our own mesoscopic model simulations [H. Zhou, M. G. Forest, and Q. Wang, J. Non-Newtonian Fluid Mech., submitted], [H. Zhou and M. G. Forest, Discrete Contin. Dyn. Syst. Ser. B, to appear]. Under these controlled conditions, we map out the kinetic phase diagram of spatiotemporal attractors of a Couette cell film in the two-parameter space of Deborah number (normalized shear rate) and Ericksen number (relative strength of elasticity potentials).
\end{abstract} flow

Key words. nematic polymer, kinetic model, structure formation, structure transition, shear

AMS subject classifications. $65 \mathrm{~N} 06,65 \mathrm{~N} 40,76 \mathrm{M} 20$

DOI. $10.1137 / 040618187$

*Received by the editors November 3, 2004; accepted for publication (in revised form) June 22, 2005; published electronically December 7, 2005. This work was sponsored by the Air Force Office of Scientific Research, Air Force Materials Command, USAF, under grants F49620-02, 1-0086, and 60088, and the National Science Foundation through grants DMI-0115445, DMS-0204243, and DMS0308019. This work was supported in part by the NASA University Research, Engineering and Technology Institute on Bio Inspired Materials (BIMat) under award NCC-1-02037.

http://www.siam.org/journals/mms/4-4/61818.html

${ }^{\dagger}$ Department of Mathematics, University of North Carolina at Chapel Hill, Chapel Hill, NC 27599 (forest@amath.unc.edu).

${ }^{\ddagger}$ Department of Mathematics and Statistics, Old Dominion University, Norfolk, VA 23529 (rzhou@ odu.edu).

$\S$ Department of Mathematical Sciences, Florida State University, Tallahassee, FL 32306 (wang@ math.fsu.edu). 
1. Introduction. Spatial distortions, clearly exposed in light scattering probes, are ubiquitous in nematic liquid crystalline polymers (LCPs) whenever processing flows are shear-dominated. These heterogeneities translate into spatially nonuniform material properties, in addition to anisotropy inherited from the orientational bias of the rod or platelet ensembles. In [13], we illustrate the effective property consequences of the unsteady morphology in the orientational distribution of rigid rod polymers presented in this paper. Property variability is typically viewed as the bane of high-performance materials technology. However, there are intriguing opportunities presented by controlled length scale "shadow zones" or "bright spots" in thermal, electrical, or mechanical response of materials, which are the near-term, applied focus of this multiscale modeling effort.

Since all film and mold-filling processes expose nematic polymers to shear-dominated flows, a quarter-century of theory, modeling, and simulation has been devoted to an understanding of sheared nematic polymers. From the nonlinear science perspective, these structure phenomena are the result of at least three coupled fields: short-range excluded-volume interactions of the macromolecules, long-range distortional elasticity of the nematic polymer liquid, and hydrodynamics. These remarks motivate the need for a solid foundation in modeling and simulation of nematic polymer processing flows.

Small-molecule liquid crystal experiments have been successfully explained by Leslie-Ericksen-Frank continuum models [3, 7]. Their macromolecular counterparts, rigid rod or platelet LCPs, exhibit much richer dynamics, more complex structure, and stronger feedback to hydrodynamics. A rich experimental history, reviewed recently by Tan and Berry [29], has guided important generalizations of continuum theory, including mesoscopic tensor models of Landau-deGennes type (cf. [3, 1, 22]), reviewed recently by Rey and Denn [26]. However, mesoscopic model predictions of LCP monodomain response versus shear rate and polymer concentration are highly model-dependent $[8,14]$; the source of sensitivity is the orientational degeneracy of quiescent nematic equilibria [25].

The key foundational step in removing the sensitivity of mesoscopic models is provided by the kinetic theory of Doi [4] and Hess [17]. Indeed, all mesoscopic tensor models can be derived from a particular closure scheme in which the kinetic Smoluchowski equation for the probability distribution function (PDF) is projected onto a dynamical system for the second-moment tensor of the PDF (cf. [27, 8, 20]). In this light, mesoscopic tensor models are revealed as a Galerkin projection onto the amplitudes of the first five spherical harmonics, giving a real five-dimensional approximation of the $\infty$-dimensional dynamics; refer to section 2 below. Recently the authors $[10,11,12,15]$ have extended seminal results of $[24,6,16]$ to compute the flowphase diagram of the Doi-Hess kinetic theory for homogeneous mesophases. These numerical studies give all attracting monodomain orientational PDFs versus nematic concentration $(N)$ and normalized shear (Peclet number $P e$ ), the so-called phase diagram. The phase diagrams for any mesoscopic tensor model can be compared with the kinetic phase diagram. One finds that no single second-moment tensor model is even qualitatively accurate except in small regions of $(N, P e)$.

The monodomain problem is the longwave limit of the structure generation problem, where one assumes homogeneous orientational distributions and a uniform imposed shear field. If mesoscopic second-moment approximations are sensitive in the longwave limit, then serious caution should be taken in mesoscopic predictions of the evolution out of the longwave regime and during structure formation. This concern has driven our emphasis toward a molecular basis for flowing nematic polymers, first for 
monodomains in linear flows, and now ([37] and this paper) for structure simulations. We extend previous one-dimensional (in physical space) structure simulations to allow for higher spherical harmonic expansions in all resolved spatial wavenumbers of the molecular orientational distribution. The numerical code and benchmark simulations are presented in [37]. Mesoscopic model simulations of structures were developed first with imposed shear kinematics [30, 31], which we follow for this paper. The authors have performed a slow plate (low Deborah number) and strong distortional elasticity (low Ericksen number) asymptotic analysis of mesoscopic models [9, 2], which reveals exact steady structures and their scaling properties. These limiting structures have been duplicated with the present kinetic model code in [37] and with mesoscopic simulations in [35] that address more general parameter regimes, although the distortional potential imposes equal elastic constants. A few mesoscopic comparisons are given in the present paper, with unequal elastic constants, to underscore the uncontrollable inaccuracy due to a Landau-deGennes mesoscopic projection of our resolved kinetic model and code.

Here we pass far out of the asymptotic limits of low Deborah and Ericksen numbers and document the structure attractors and transitions for imposed flow. These numerical continuation studies lay the foundation for passage to distortions with extremely high Ericksen numbers (physically plausible values are on the order of tens of thousands) and with confinement boundary conditions, where the length scale spectrum of morphology is predicted to scale like $\mathrm{Er}^{-p}$, with $0<p<1$. The infinite Ericksen number limit decouples the boundary conditions and gradient morphology, and then highly sensitive monodomain responses are the challenge instead of structure formation. Thus, the present study gives one more interpolation point toward realistic processing parameter regimes. We also derive mesoscopic, second-moment tensor approximate models and present illustrative simulations at intermediate Ericksen number $(E r=500)$; we then compare the Deborah number cascades in structure attractors for the full kinetic code and two closure schemes.

The extension to flow feedback in multiparameter kinetic simulations, already illustrated in [37], will be reported in subsequent communications, along with release of the molecular distribution out of the shear plane (in orientational space). We note that two-dimensional, physical space structure simulations have been performed by Leal and coworkers [28], using a mesoscopic projection of the model presented here. Our philosophy is to establish a foundation of multiscale theory and simulations starting from simple, controlled assumptions on the orientational and hydrodynamic degrees of freedom and spatial dimensions. We then release physical and orientational space restrictions and explore persistent versus new consequences. Our extension to higher physical space dimensions remains for future communications.

2. Governing multiscale equations for sheared LCP films. We consider plane shear flow of an ensemble of high aspect ratio, rigid rods in a viscous solvent, confined between two parallel plates located at $y= \pm h$, in Cartesian coordinates $\mathbf{x}=(x, y, z)$ and moving with corresponding velocity $\mathbf{v}=\left( \pm v_{0}, 0,0\right)$, respectively. The rods are modeled as spheroids with axis of symmetry, $\mathbf{m}$, whose PDF in time and across the shear gap is our primary focus. We will give the kinetic description of this PDF and then the standard second-moment truncation, which requires a choice of closure scheme.

There are two apparent length scales in this problem: the gap width $2 h$, an external length scale, and the finite range $l$ of molecular interaction, an internal length scale, set by the distortional elasticity in the Doi-Marrucci-Greco (DMG) 
model. When the plates move relative to each other at a constant speed, it sets a bulk flow time scale $\left(t_{0}=\frac{h}{v_{0}}\right)$; the nematic average rotary diffusivity $\left(D_{r}^{0}\right)$ sets another (internal) time scale $\left(t_{n}=\frac{1}{D_{r}^{0}}\right)$, and the ratio $t_{n} / t_{0}$ defines the Deborah number $D e$. There are also scales associated with solvent viscosity and the three nematic viscosities, and with elastic distortion, which due to the flow-nematic-plate interaction, are not a priori known.

We nondimensionalize the DMG model using the length scale $h$, the time scale $t_{n}$, and the characteristic stress $\tau_{0}=\rho h^{2} / t_{n}^{2}$. The dimensionless flow and stress variables become

$$
\tilde{\mathbf{v}}=\frac{t_{n}}{h} \mathbf{v}, \quad \tilde{\mathbf{x}}=\frac{1}{h} \mathbf{x}, \quad \tilde{t}=\frac{t}{t_{n}}, \quad \tilde{\tau}=\frac{\tau}{\tau_{0}}, \quad \tilde{p}=\frac{p}{\tau_{0}} .
$$

The following seven dimensionless parameters arise:

$$
R e=\frac{\tau_{0} t_{n}}{\eta}, \quad \alpha=\frac{3 c k T}{\tau_{0}}, \quad E r=\frac{8 h^{2}}{N l^{2}}, \quad \mu_{i}=\frac{3 c k T \zeta_{i} t_{0}}{t_{n} \tau_{0}}, i=1,2,3, \quad \theta=\frac{L^{2}}{\mathcal{L}^{2}},
$$

where $R e$ is the solvent Reynolds number; $\alpha$ measures the strength of entropic relative to kinetic energy; Er is the Ericksen number which measures short-range nematic potential strength relative to distortional elasticity strength depicted by the persistence length $l ; \mu_{i}, i=1,2,3$, are three nematic Reynolds numbers; and $\theta$ is a fraction between 0 and 1 that corresponds to equal $(\theta=0)$ or distinct $(\theta \neq 0)$ elasticity constants $[32,2]$. For other parameters, we refer to [32].

We drop the tilde on all variables; all figures correspond to normalized variables and length and time scales. We neglect the effect of translational diffusion and employ an approximate rotary diffusivity [5].

2.1. Kinetic theory for the rigid rod, viscous solvent dispersion. The dimensionless Smoluchowski equation for the probability $\operatorname{PDF} f(\mathbf{m}, \mathbf{x}, t)$ becomes

$$
\begin{aligned}
& \frac{D f}{D t}=\mathcal{R} \cdot[(\mathcal{R} f+f \mathcal{R} V)]-\mathcal{R} \cdot[\mathbf{m} \times \dot{\mathbf{m}} f], \\
& \dot{\mathbf{m}}=\Omega \cdot \mathbf{m}+a[\mathbf{D} \cdot \mathbf{m}-\mathbf{D}: \mathbf{m m m}],
\end{aligned}
$$

where $D / D t$ is the material derivative $\partial / \partial t+\mathbf{v} \cdot \nabla, \mathcal{R}$ is the rotational gradient operator

$$
\mathcal{R}=\mathbf{m} \times \frac{\partial}{\partial \mathbf{m}}
$$

$\mathbf{D}$ and $\Omega$ are the symmetric and antisymmetric parts of $\nabla \mathbf{v}$, and $a=\left(r^{2}-1\right) /\left(r^{2}+1\right)$ is the molecular shape parameter for spheroidal macromolecules of aspect ratio $r$. The Doi-Marrucci-Greco potential, coupling a homogeneous Maier-Saupe excluded volume interaction together with anisotropic distortional elasticity, is

$$
V=-\frac{3 N}{2}\left[\left(\mathbf{I}+\frac{1}{3 E r} \Delta\right) \mathbf{M}: \mathbf{m m}+\frac{\theta}{3 E r}(\mathbf{m m}:(\nabla \nabla \cdot \mathbf{M}))\right],
$$

where the second moment tensor $\mathbf{M}$ of $f$ is

$$
\mathbf{M}=\mathbf{M}(f)=\int_{\|\mathbf{m}\|=1} \mathbf{m m} f(\mathbf{m}, \mathbf{x}, t) d \mathbf{m} .
$$


The dimensionless forms of the balance of linear momentum, the stress constitutive equation, and the continuity equation are (cf. [32])

$$
\begin{aligned}
\frac{d \mathbf{v}}{d t}= & \nabla \cdot(-p \mathbf{I}+\tau) \\
\tau= & \left(\frac{2}{R e}+\mu_{3}(a)\right) \mathbf{D}+a \alpha\left(\mathbf{M}-\frac{\mathbf{I}}{3}-N \mathbf{M} \cdot \mathbf{M}+N \mathbf{M}: \mathbf{M}_{4}\right) \\
& -a \frac{\alpha}{6 E r}\left(\Delta \mathbf{M} \cdot \mathbf{M}+\mathbf{M} \cdot \Delta \mathbf{M}-2 \Delta \mathbf{M}: \mathbf{M}_{4}\right) \\
& -\frac{\alpha}{12 E r}(2(\Delta \mathbf{M} \cdot \mathbf{M}-\mathbf{M} \cdot \Delta \mathbf{M})+(\nabla \mathbf{M}: \nabla \mathbf{M}-(\nabla \nabla \mathbf{M}): \mathbf{M})) \\
& -a \frac{\alpha \theta}{12 E r}\left[\mathbf{M} \cdot \mathbf{M}_{d}+\mathbf{M}_{d} \cdot \mathbf{M}-4(\nabla \nabla \cdot \mathbf{M}): \mathbf{M}_{4}\right] \\
& -\frac{\alpha \theta}{12 E r}\left[\mathbf{M}_{d} \cdot \mathbf{M}-\mathbf{M} \cdot \mathbf{M}_{d}-(\nabla \nabla \cdot \mathbf{M}) \cdot \mathbf{M}+\mathbf{M}_{\beta j, \alpha} \mathbf{M}_{i j, i}\right] \\
& +\left[\mu_{1}(a)(\mathbf{D} \cdot \mathbf{M}+\mathbf{M} \cdot \mathbf{D})+\mu_{2}(a) \mathbf{D}: \mathbf{M}_{4}\right], \\
\nabla \cdot \mathbf{v}= & 0
\end{aligned}
$$

where $\mathbf{M}_{d}$ is a symmetrized second variation of $\mathbf{M}$ and $\mathbf{M}_{4}$ is the fourth moment of $f$,

$$
\mathbf{M}_{d}=\nabla \nabla \cdot \mathbf{M}+(\nabla \nabla \cdot \mathbf{M})^{T}, \quad \mathbf{M}_{4}=\int_{\|\mathbf{m}\|=1} \operatorname{mmmm} f(\mathbf{m}, \mathbf{x}, t) d \mathbf{m} .
$$

Rheological properties of nematic LCPs are gleaned from $\tau$ in (7) and are depicted below in several kinetic simulations.

2.2. Mesoscopic closure models. Mesoscopic models replace the Smoluchowski equation (3) with a closed dynamical system for the second moment $\mathbf{M}$ and approximate the stress (7) by closure expressions for terms involving the fourth moment $\mathbf{M}_{4}$. We summarize the procedure below and give a class of Landau-deGennes mesoscopic models. These models form the basis for numerical comparison of structure generation between our resolved PDF code and second-moment truncated models.

We apply the following parametrized closure to approximate terms involving the fourth moment $\mathbf{M}_{4}=\langle\mathbf{m m m m}\rangle$, defined in (8), in terms of second moments:

$$
\mathbf{A}: \mathbf{M}_{4}=c \mathbf{A}: \mathbf{M} \mathbf{M}+\frac{1}{2}(1-c)(\mathbf{A} \cdot \mathbf{M}+\mathbf{M} \cdot \mathbf{A})
$$

where $\mathbf{A}$ is an arbitrary, symmetric second-order tensor and $c$ is an interpolation parameter. Taking the second moment of the Smoluchowski equation (3) and applying the above closure twice, once for $\mathbf{A}=\mathbf{M}$ with $c=c_{1}$ and once for $\mathbf{A}=\mathbf{D}$ with $c=c_{2}$, we obtain the self-consistent mesoscopic model:

$$
\begin{aligned}
& \frac{D \mathbf{M}}{D t}-\Omega \cdot \mathbf{M}+\mathbf{M} \cdot \Omega-a c_{2}[\mathbf{D} \cdot \mathbf{M}+\mathbf{M} \cdot \mathbf{D}] \\
= & -2 a c_{2} \mathbf{D}: \mathbf{M M}-6\left[\mathbf{M}-\frac{\mathbf{I}}{3}-c_{1} N \mathbf{M}^{2}+c_{1} N \mathbf{M}: \mathbf{M M}\right. \\
& -\frac{c_{1}}{6 E r}(\Delta \mathbf{M}: \mathbf{M}+\mathbf{M}: \Delta \mathbf{M}-2 \Delta \mathbf{M}: \mathbf{M M})+\frac{\theta}{E r}(\nabla(\nabla \cdot \mathbf{M}) \cdot \mathbf{M} \\
& \left.\left.+(\nabla(\nabla \cdot \mathbf{M}) \cdot \mathbf{M})^{T}+\left((\nabla \nabla \cdot \mathbf{M})^{T} \cdot \mathbf{M}\right)+\mathbf{M} \cdot \nabla \nabla \cdot \mathbf{M}-4 \mathbf{M}:(\nabla \nabla \cdot \mathbf{M}) \mathbf{M}\right)\right] .
\end{aligned}
$$

For $c_{1}=c_{2}=1$, we recover the Doi closure model equation, which for equal elasticity constants $(\theta=0)$ forms the basis of our previous structure studies $[9,34,35,36]$. We 
also select $c_{1}=0.6$ in simulations reported below so that the critical concentration, $N_{c}=5$, where the isotropic phase loses stability in the mesoscopic closure model, matches the kinetic theory result; the same rule is enforced for the second parameter, so that $c_{2}=c_{1}$.

Traditionally, the second moment $\mathbf{M}$ is replaced by its deviatoric part $\mathbf{Q}$ (a secondorder, symmetric, traceless tensor) known as the orientation tensor (or structure tensor):

$$
\mathbf{Q}=\mathbf{M}-\frac{1}{3} \mathbf{I}
$$

2.3. Boundary and initial conditions. We consider one-dimensional physical space (the interval between the two parallel plates). The boundary conditions on the velocity $\mathbf{v}=\left(v_{x}, 0,0\right)$ are given by the Deborah number

$$
v_{x}(y= \pm 1, t)= \pm D e .
$$

Following previous studies [3, 21, 31, 9], we assume homogeneous anchoring at the plates, given by the quiescent nematic equilibrium,

$$
f(\mathbf{m}, y= \pm 1, t)=f_{e}(\mathbf{m}),
$$

where $f_{e}(\mathbf{m})$ is an equilibrium solution of (3) with $\mathbf{v}=\mathbf{0}$. At nematic concentrations, equilibria $f_{e}(\mathbf{m})$ are invariant under orthogonal rotations; orientational degeneracy is broken in the laboratory by mechanical rubbing or applied fields. In this study, we assume tangential and normal anchoring; that is, the peak orientation (so-called major director) on the boundary is aligned with the plate motion axis or normal to it. The initial condition for the PDF is given by

$$
f(\mathbf{m}, y, t=0)=f_{e}(\mathbf{m}) ;
$$

that is, the molecular liquid at the start of the simulation is in a statistically uniform, thermal equilibrium, with the degeneracy of the peak axis following the plate anchoring condition.

2.4. The numerical algorithm. We briefly review the numerical methods for the kinetic equation from [37]. We seek a solution of the PDF in a spherical harmonic representation:

$$
f(\mathbf{m}, \mathbf{x}, t) \approx \sum_{l=0}^{L} \sum_{m=-l}^{l} a_{l}^{m}(\mathbf{x}, t) Y_{l}^{m}(\mathbf{m}) .
$$

We then apply a standard Galerkin scheme to arrive at a system of 65 coupled, nonlinear partial differential equations for $a_{l}^{m}$, corresponding to the truncation order $L=10$ (for in-plane simulations, only 35 equations with even $m$ are needed). Spatial derivatives are discretized using fourth-order finite difference methods, and an adaptive moving mesh algorithm is important for efficiency and to capture localized internal and boundary layers with strong defocusing of the PDF. Spectral deferred corrections are used for time integration to achieve fourth-order convergence and thereby remove dynamic sensitivity especially near transition phenomena.

For the mesoscopic model, the algorithm is similar except that we solve only for the components of the second-moment tensor $\mathbf{Q}$ (a reduction from 35 to 3 components for confined in-plane simulations and from 65 to 5 for general out-of-plane orientation simulations). 
TABLE 1

In-plane structure attractors and phase transitions for three decades of Deborah number (De) and Ericksen number (Er). ES and VS stand for elastic (E) and viscous (V) dominated steady (S) states. T or $W$ indicates a transient structure in which the peak orientation axis at each height between the plates either oscillates with finite amplitude (wagging) or rotates continuously (tumbling).

\begin{tabular}{|c||c|c|c|c|c|c|c|c||c|}
\hline \hline$D e \backslash E r$ & 5 & 10 & 15 & 50 & 180 & 500 & 2000 & 5000 & $\infty$ \\
\hline \hline 0.01 & ES & ES & ES & ES & ES & ES & TW & TW & T \\
\hline 0.05 & ES & ES & ES & ES & ES & TW & TW & TW & T \\
\hline 0.1 & ES & ES & ES & ES & W & TW & TW & TW & T \\
\hline 0.5 & ES & ES & ES & W & TW & TW & TW & TW & T \\
\hline 1 & ES & ES & W & TW & TW & TW & TW & TW & T \\
\hline 3 & ES & ES & $\mathbf{W}$ & TW & TW & TW & TW & TW & T \\
\hline 5.5 & ES & ES & W & TW & TW & TW & TW & TW & T \\
\hline 6 & ES & ES & W & W & W & W & W & W & W \\
\hline 8 & ES & ES & W & W & W & W & W & W & W \\
\hline 8.5 & ES & ES & ES & VS & VS & VS & VS & VS & FA \\
\hline 10 & ES & ES & ES & VS & VS & VS & VS & VS & FA \\
\hline 12 & ES & ES & VS & VS & VS & VS & VS & VS & FA \\
\hline \hline
\end{tabular}

3. Structure formation-kinetic simulations. In all numerical computations in this paper, we fix a nematic concentration $N=6$ and consider structure attractors versus the Ericksen number $E r$ and Deborah number De for two different anchoring conditions. Other parameters we use in the simulation include $\alpha=2$, $\theta=0.5, \mu_{1}=0.0004, \mu_{2}=0.15$, and $\mu_{3}=0.01$. Four distinct spatiotemporal attractors arise and are listed in Table 1 . The remainder of the paper is a discussion of the content of Table 1. These attractors are qualitatively consistent with the mesoscopic model simulations of Tsuji and Rey [30,31]; the closure rules which produce their model are given in [8]. The boundaries between attractor types are captured on a coarse parameter grid, giving the structure attractor analogue of monodomain attractor phase diagrams (cf. $[8,10,11])$. Our purpose for this paper is to illustrate each attractor type and amplify its rheological signature, and then use this structure phase diagram (Table 1) to identify the significance of structure phase transitions.

3.1. Elasticity-dominated steady states (ES structure attractors). When spatial elasticity dominates, i.e., the Ericksen number is low, together with moderate plate speeds (Deborah number not huge), the elastic potential overwhelms the viscous forces. In such cases, the material saturates in a steady structure whose stored elastic stresses balance the viscous stress. To view the transient and steady state anisotropy in the film, Figures 1-3 focus on the peak orientation angle $\phi$ of the PDF, associated with the so-called major director of the nematic liquid. This PDF feature and subsequent properties related to the shape of the distribution are computed in postprocessing from the kinetic simulation as follows. We first grab the first five spherical harmonic amplitudes to form the second-moment tensor $\mathbf{M}(f)$, defined in (6), then calculate the distinguished eigenvector (major director)

$$
\mathbf{n}_{1}=(\cos \phi, \sin \phi, 0)
$$




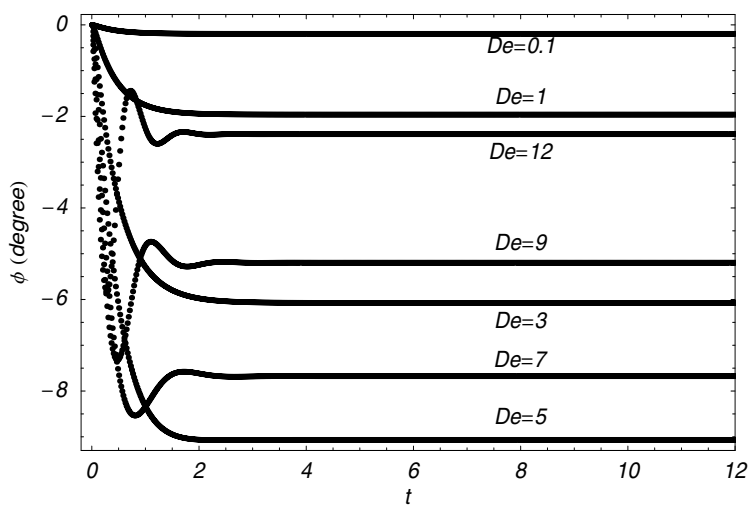

FIG. 1. Evolution of the peak orientation angle midway between the two plates for $E r=5$ and two decades of De, with parallel anchoring at the plates $(\phi=0)$. The strong distortional elasticity at low Er leads to steady structure attractors with a robust time scale of convergence to steady state indicated by arrested motion at the midplane.
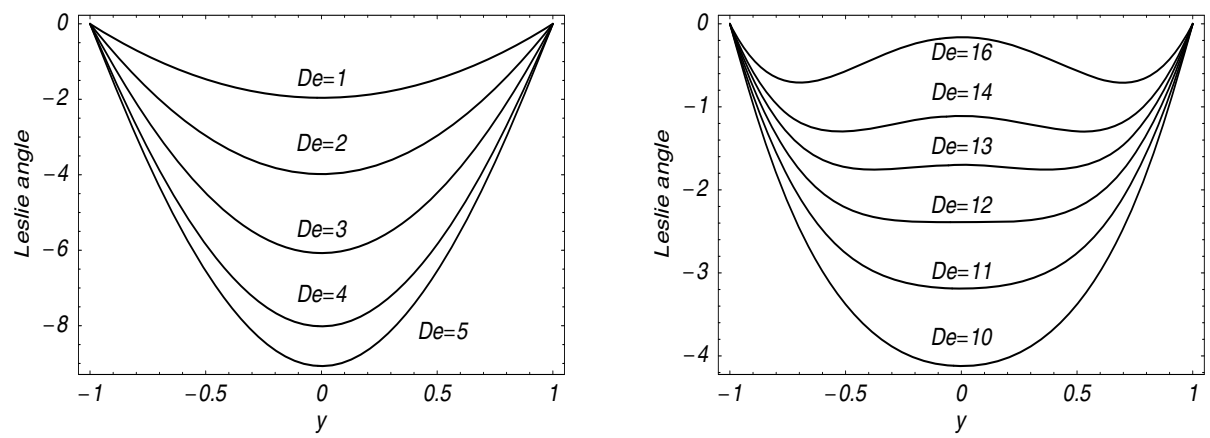

FIG. 2. Steady states for fixed small Ericksen number $E r=5$ and increasing Deborah number with parallel anchoring. Left: De $=1,2,3,4,5$. Right: De $=10,11,12,13,14,16$.

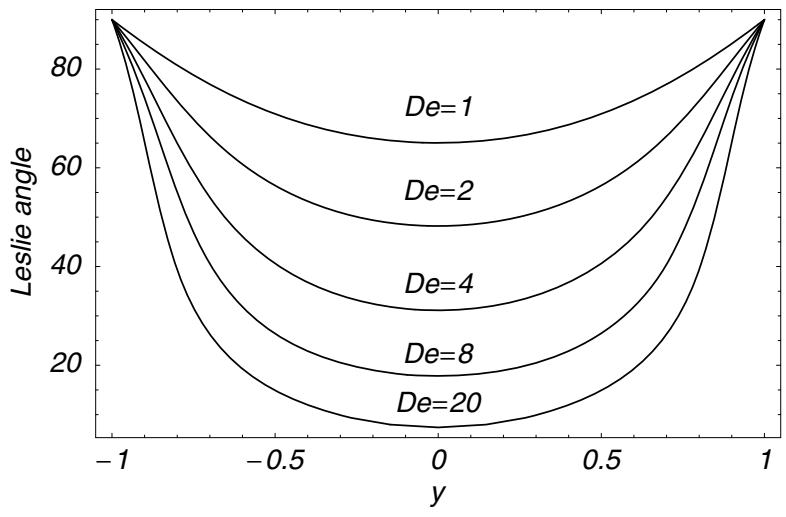

FIG. 3. Steady states for fixed small Ericksen number $E r=5$ and increasing Deborah number, De $=1,2,4,8,20$, with normal anchoring.

associated with the largest eigenvalue, $d_{1}$, where $0 \leq d_{3} \leq d_{2} \leq d_{1} \leq 1$ are eigenvalues of $\mathbf{M}$, and $\mathbf{n}_{1,2,3}$ are the corresponding orthonormal basis of eigenvectors. Clearly, 
$\mathbf{n}_{1}$ is uniquely defined if and only if $d_{1}$ is a simple eigenvalue; otherwise, the associated orientational distribution is considered a defect, as amplified below. The comparable shape distortions of the PDF are detected by this largest eigenvalue $d_{1}$. We refer to [37] for additional details.

Figure 1 focuses on the evolution of the peak orientation angle $\phi$ at the center of the plate gap, for $E r=5$ and two decades of $D e$, with parallel anchoring. The midplane orientation angle converges monotonically to steady state at sufficiently low $D e$, then acquires an oscillatory transient for $D e>5$. This steady angle is called the Leslie alignment angle, by analogy with Leslie-Ericksen director theory. Note the remarkably robust convergence time of 2 units (twice the mean molecular relaxation time of the nematic liquid) at the midplane of the cell. Not shown here is the corresponding peak orientation axis across the gap, which reveals the arrested motion of the major director as a wave that sweeps from the plates, where $\phi=0$ is pinned by the anchoring condition. As time $t$ approaches 2 , for all $D e$ shown, the interior transient layer shrinks until finally the entire gap is steady. These elasticity (E) dominated steady (S) states are labeled ES, following Tsuji and Rey [31].

We turn now to the steady state structure across the gap and defer the space-time transient properties to more interesting regimes. Figure 2 illustrates a $D e$-dependent continuation study of gap heterogeneity in the Leslie alignment angle for $E r=5$, with parallel anchoring. Figure 3 shows the analogous results for normal anchoring. Clearly, there is a dominant parabolic gap structure at low $D e$ for both anchoring conditions, and then new length scales enter for higher $D e$. These kinetic properties confirm mesoscopic model analysis and numerical continuation studies in [35, 9, 2]. These peak axis distortions of the PDF are referred to as nematic elasticity. We note that in the longwave limit, with $E r=\infty$, the in-plane response at low to moderate $D e$ is tumbling. But, with this strong spatial coupling through distortional elasticity for $E r=5$, the anchoring at the plates propagates inward, resulting in arrested tumbling [23] across the entire gap. For high $D e$, the monodomain response is flow-aligning, so one expects a steady structure as $D e$ increases that essentially interpolates between the boundary anchoring condition and the monodomain Leslie alignment angle for that $D e$. This indeed occurs, as indicated in Table 1, though we omit the details of transient approach to the steady structure.

Next, we focus on the shape distortions of the PDF, or so-called molecular elasticity, measured by the order parameter $d_{1}$. Recall that an isotropic PDF obeys $f \equiv 1 /(4 \pi), d_{1}=d_{2}=d_{3}=1 / 3$, so that larger values of $d_{1}$ reflect focusing of the PDF around the peak axis $\mathbf{n}_{1}$. Some authors use $d_{1}-d_{2}$, the Flory order parameter, whose departure from zero measures relative focusing of the PDF between the primary $\left(\mathbf{n}_{1}\right)$ and secondary $\left(\mathbf{n}_{2}\right)$ axes of the second moment $\mathbf{M}(f)$. We use Figure 4 to convey a representative shape-distortion profile across the gap, with $E r=5$ and $D e=2$ from Figures 2 and 3. The large value of $d_{1}$ across the gap signals a highly focused PDF. The distortions are minor with parallel anchoring and correlate with the nematic director structure, whereas normal anchoring leads to a new length scale in the PDF. Note with parallel anchoring the PDF weakly defocuses from the plate to the midgap, whereas normal anchoring has peak focusing at the center of the gap. (These features are important for material properties, since the principal values of conductivity in a nematic polymer composite are directly correlated with the principal values $d_{i}$ of $\mathbf{M}(f)$; see [33].)

ES attractors persist for moderate $E r$ and low $D e$, e.g., $E r=500, D e=0.01$, but convergence to these steady structures is extremely slow. Figure 5 shows the slow evolution of the alignment angle in the midgap and the final steady state structure. An 

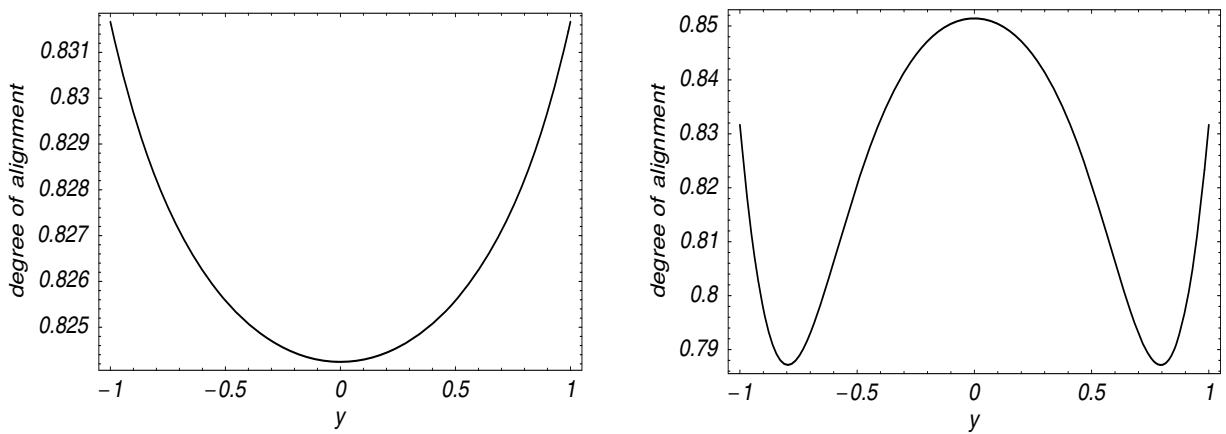

FIG. 4. The degree of alignment of LCPs in the steady state for $E r=5, D e=2$. Left: parallel anchoring. Right: normal anchoring.
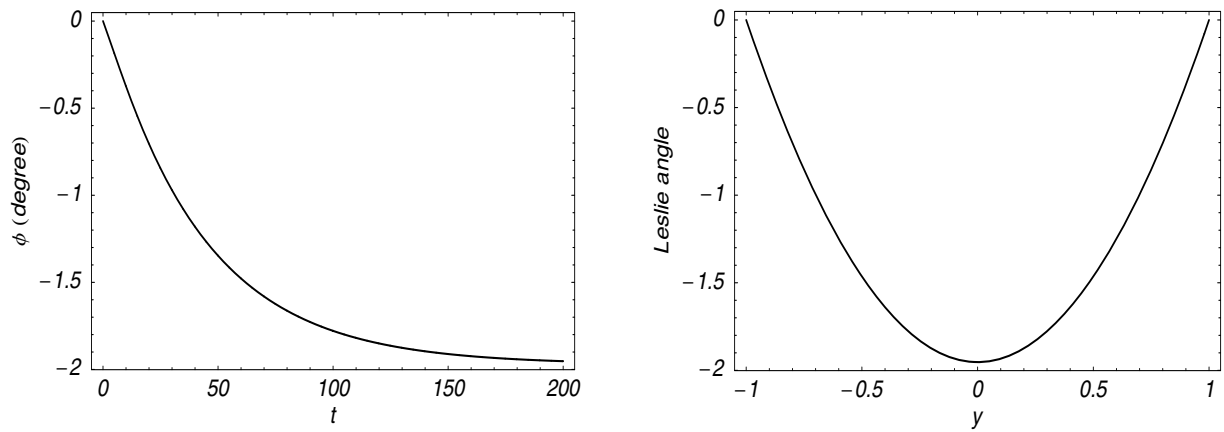

FIG. 5. Left: evolution of the director alignment angle of LCPs at the midgap for $E r=500$, $D e=0.01$. Right: Leslie alignment angle across the gap in the steady state for the same parameters Er and De.

overall observation of ES attractors is that structure features are smoothly dependent on $\mathrm{De}$ and $\mathrm{Er}$.

3.2. Viscous-dominated steady states (VS structure attractors). For sufficiently high Deborah number, the viscous driving forces induced by the moving plates will overwhelm short-range elasticity (which governs bulk monodomain dynamics), and the molecular distribution at each gap height aligns at some preferred direction. The spatial elasticity potential mediates the plate anchoring angle and the bulk preferred PDF. An example of a VS attractor is shown in Figure 6. On the left, the solid line shows the evolution of the peak orientation angle in the middle of the gap for $E r=1000, D e=10$, starting from equilibrium with parallel anchoring. For comparison, we also show a monodomain simulation, i.e., with $E r=\infty$, for which the corresponding time evolution of the alignment angle is the dotted curve in this figure. The dots are coincident with the solid line, meaning that for these conditions the midgap LCP liquid does not sense the presence of the plates. This is further reflected in the structure properties; a nearly uniform, long wavelength interior layer that spans most of the gap, during the evolution (Figure 6, right) and in the steady attractors (Figure 7) for tangential and normal anchoring. This steady structure, in which spatial distortions are confined in plate boundary layers, is consistent with analytical predictions from mesoscopic tensor models at low $D e$ and $\operatorname{Er}[9,2]$. These figures show that the boundary layer structures include comparable variations in the peak axis $(\phi)$ 

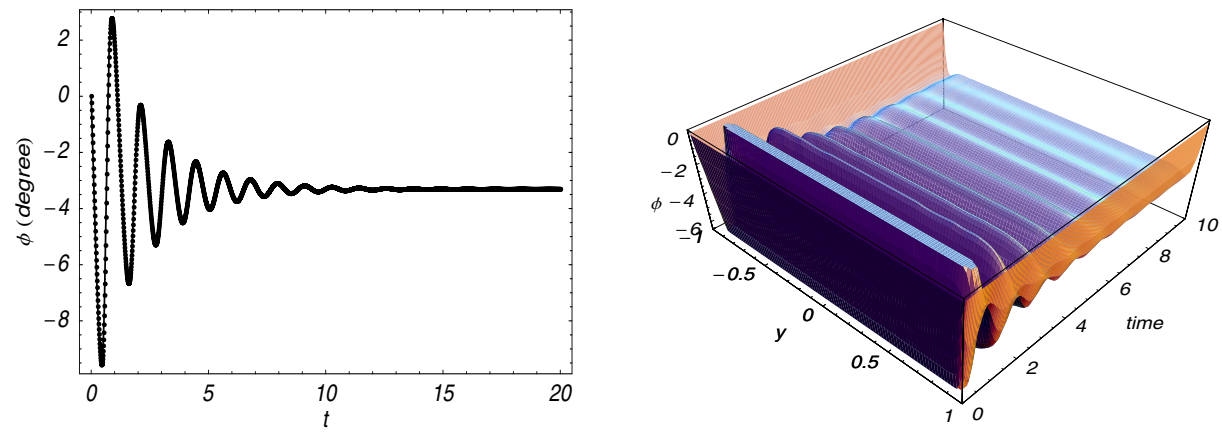

FIG. 6. Left: evolution of the peak PDF alignment angle of LCPs in the middle of the gap for $E r=1000, D e=10$ with parallel anchoring. Right: evolution of the peak alignment angle of the PDF across the gap, showing dynamics of a coherent spatial structure.
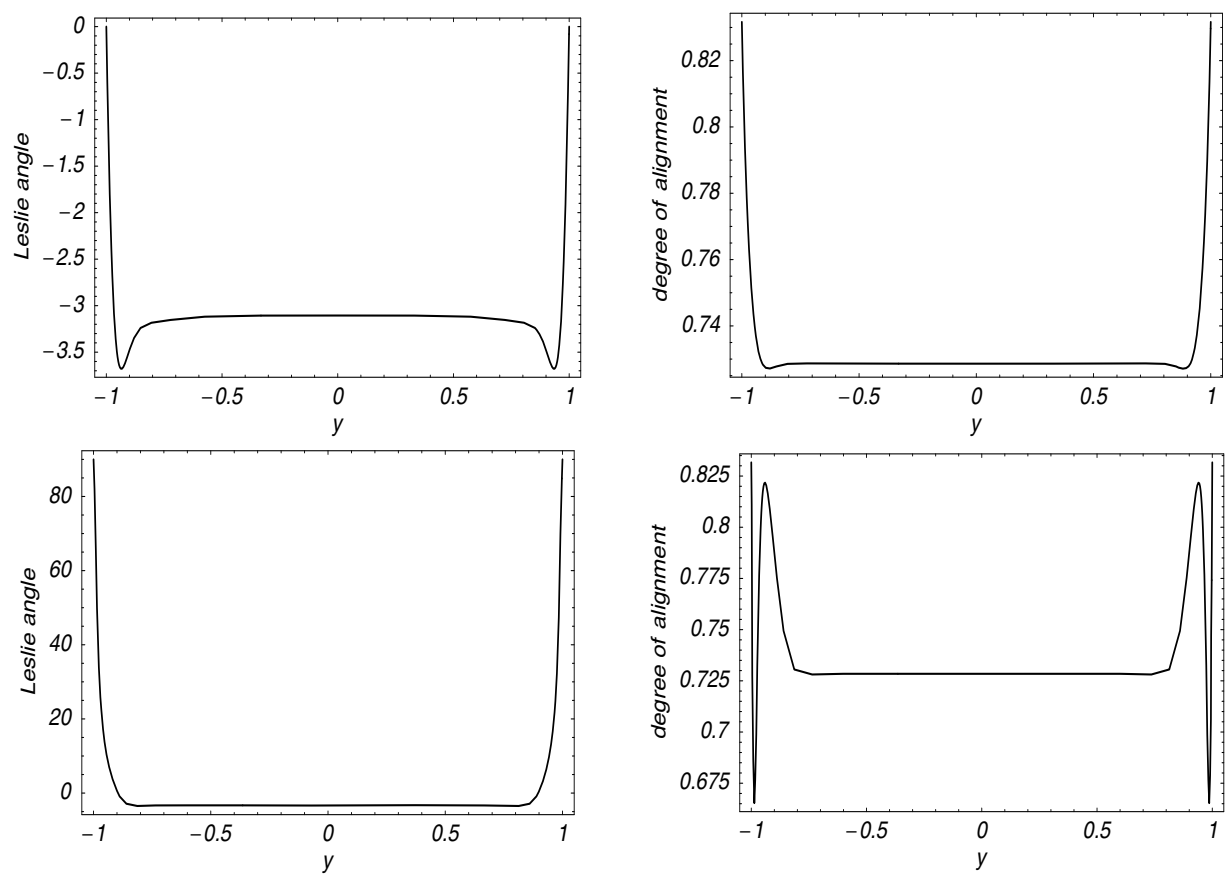

FIG. 7. Leslie alignment angle and degree of alignment extracted from the PDF of a VS attractor for $E r=1000, D e=10$. Top: parallel anchoring. Bottom: normal anchoring.

and shape $\left(d_{1}\right)$ of the PDF for both anchoring conditions. However, the amplitude distortions in $\phi$ and $d_{1}$ in the boundary layers are much greater for normal anchoring conditions, since the midgap Leslie alignment angle $\phi_{L} \approx-3^{\circ} \approx-0.05$ radians is significantly conflicted with the normal anchoring angle $\phi \approx 90^{\circ}$ or $\pi / 2$ radians. The oscillations in Figure 6 are consistent with simulations in [31] using second-moment model equations. The sharp boundary layers (Figure 7) in the VS attractors for both parallel and normal anchoring provide a distinction with ES structure attractors (Figures 2 and 3 ). 

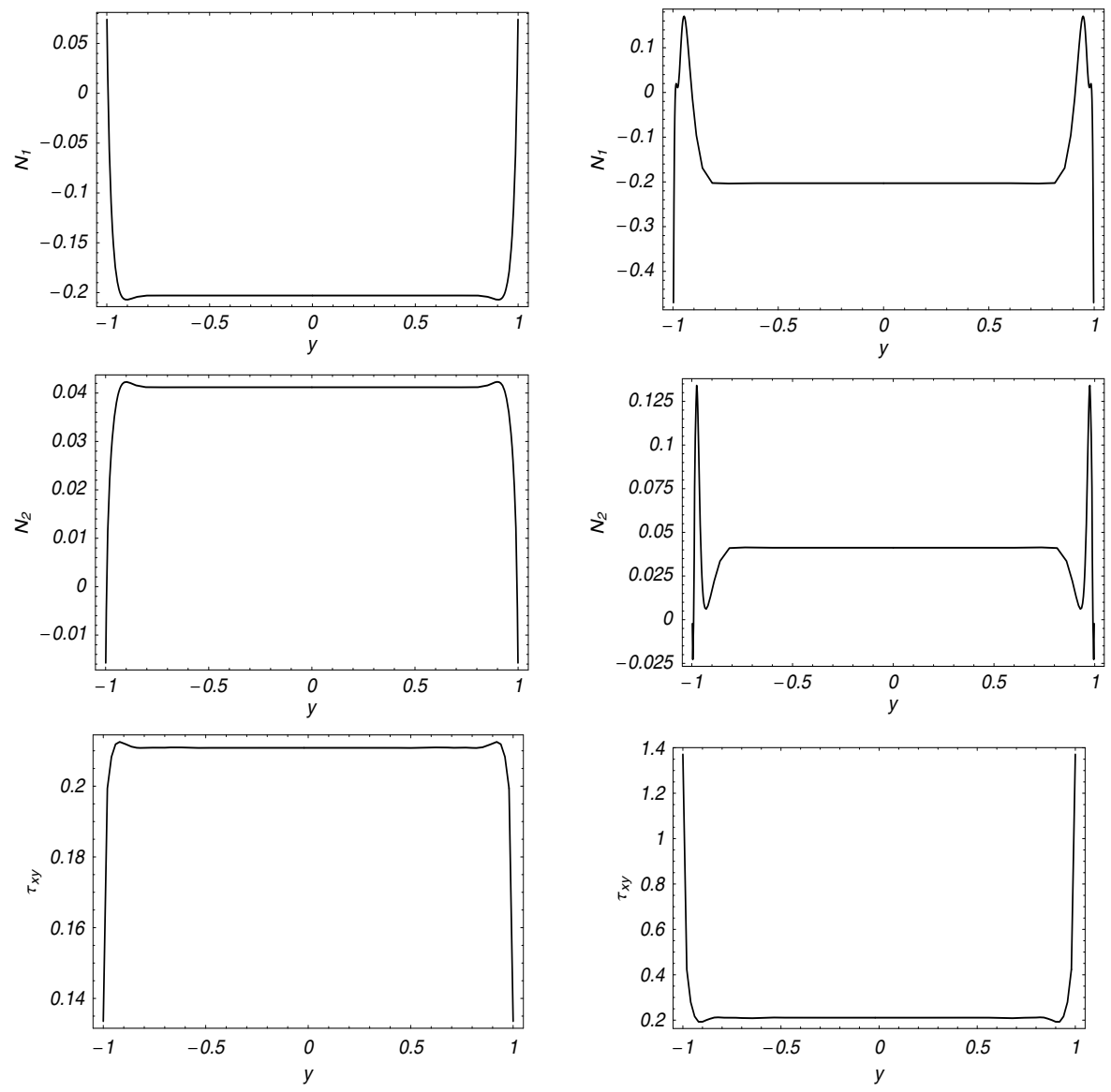

FIG. 8. Normal stress differences $N_{1}, N_{2}$ and shear stress $\tau_{x y}$ of VS structures for $E r=1000$, De $=10$. Left: parallel anchoring. Right: normal anchoring.

Figure 8 shows the normal stress differences

$$
N_{1}=\tau_{x x}-\tau_{y y}, \quad N_{2}=\tau_{y y}-\tau_{z z}
$$

and the shear stress $\tau_{x y}$ for the attractors of Figures 6 and 7 . Except in the boundary layers, the first normal stress difference $\left(N_{1}\right)$ is negative, while the second normal stress difference $\left(N_{2}\right)$ is positive. These data are directly measurable in experiments; the sign of $N_{1}$, for example, determines whether the liquid is drawing the plates toward one another or pushing them apart. Sign changes within the sample require an average over the gap to determine effective normal stress differences. This rheological feature also has strong implications for mechanical properties once a nematic polymer film has been quenched, representing stored residual stresses. These results are consistent with monodomain kinetic simulations in [11] for the same concentration $N=6$ and Deborah number $D e=10$. The sharp boundary layers appear in all stress profiles in Figure 8. Note that in the middle of the plates, all stress data are basically independent of boundary anchoring, consistent with the longwave uniform structure that consumes the middle $75-80 \%$ of the gap.

Next we explore the steady state transition from ES to VS attractors at high Deborah number, $D e=12$, by varying the Ericksen number; see Figure 9 (left). Results 

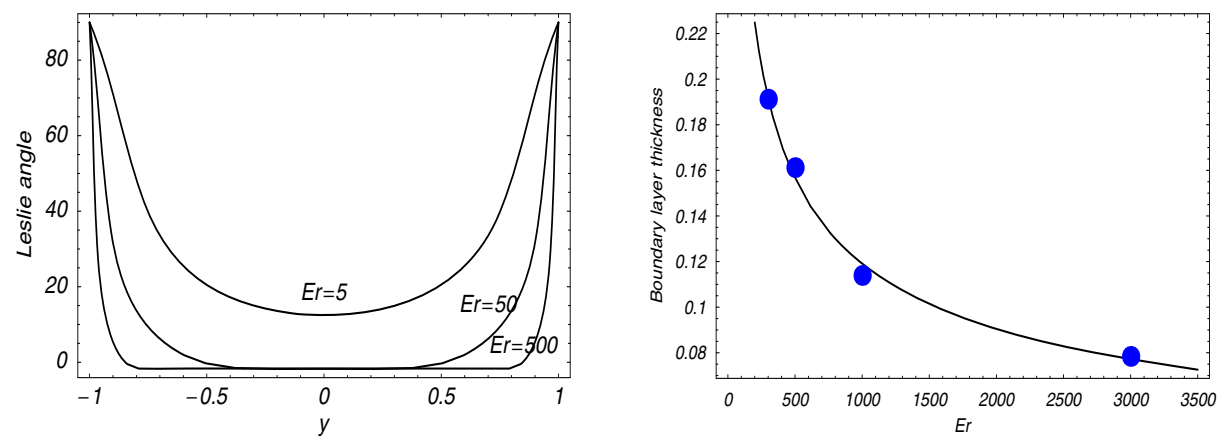

FIG. 9. Left: structure continuation profiles with fixed De $=12$ and varying Er, for normal anchoring. The nematic structure features of ES attractors at low Er develop sharp boundary layer features at high Er, typical of VS attractors. Right: the scaling behavior of the boundary layer thickness. The solid line shows the exponential fit of the discrete data (dots).

from normal anchoring are chosen. For small Ericksen number with distortional elasticity dominant, the alignment angle structure is parabolic-like. By increasing $E r$ while holding $D e$ fixed (achieved by shortening the persistence length $l$ of Frank elasticity), sharp boundary layers emerge. The scaling behavior of the boundary layer thickness in the asymptotic limit of low $D e$ and $E r$ is $E r^{-1 / 2}$ based on mesoscopic model analysis [2,9]. For $D e=12$, the behavior is shown in Figure 9 (right). To produce this figure, we calculate the gradient of the alignment angle in the gap. In the boundary layer, the gradient is large, while in the interior bulk region, it is near zero. We record the transition point in the director gradient, choosing an arbitrary value of order 1 or 10, and use this diagnostic to estimate the thickness of the boundary layer. The best exponential fit from this data suggests a boundary layer width scaling behavior of approximately $E r^{-0.4}$, which is consistent within this crude characterization with the $E r^{-1 / 2}$ asymptotic scaling of mesoscopic models. This result speaks to the value of analytical approximations in providing guides for scaling behavior. As $E r \rightarrow \infty$, we get the limit case where the entire gap exhibits the same response.

3.3. Composite tumbling-wagging periodic states ( $T W$ structure attractors). The steady attractors ES and VS give way to spatiotemporal attractors when neither elasticity nor viscosity dominates. These attractor properties can be anticipated as follows. The PDF is anchored at the plates; absent of spatial elasticity, the homogeneous response to moderate shear rate $(D e=O(1))$ involves tumbling of the peak axis of the PDF. So, for moderate $E r$, one expects tumbling $(T)$ at interior locations with a slight phase shift between neighboring height locations; this implies an interior tumbling layer with slight spatial modulations across the layer. At some height, the monotone director tumbling transitions to a finite oscillation, called wagging $(\mathrm{W})$. A wagging layer then persists, with amplitude of oscillation decreasing to zero as the plate is approached. These structure phenomena occur for sufficiently large $E r$ and moderate $D e$. An example is shown in Figure 10 for $E r=500$ and $D e=4$ with parallel anchoring. The left-hand figure shows evolution of the PDF peak alignment angle in the middle of the gap, which oscillates between $-90^{\circ}$ and $90^{\circ}$, meaning continuous rotation of the peak of the PDF, while the righthand figure shows the peak alignment angle near the plates, which oscillates with amplitude less than $90^{\circ}$, moving from the plates inward. The wagging amplitudes increase to $90^{\circ}$, creating a transition location between the wagging layer and the 

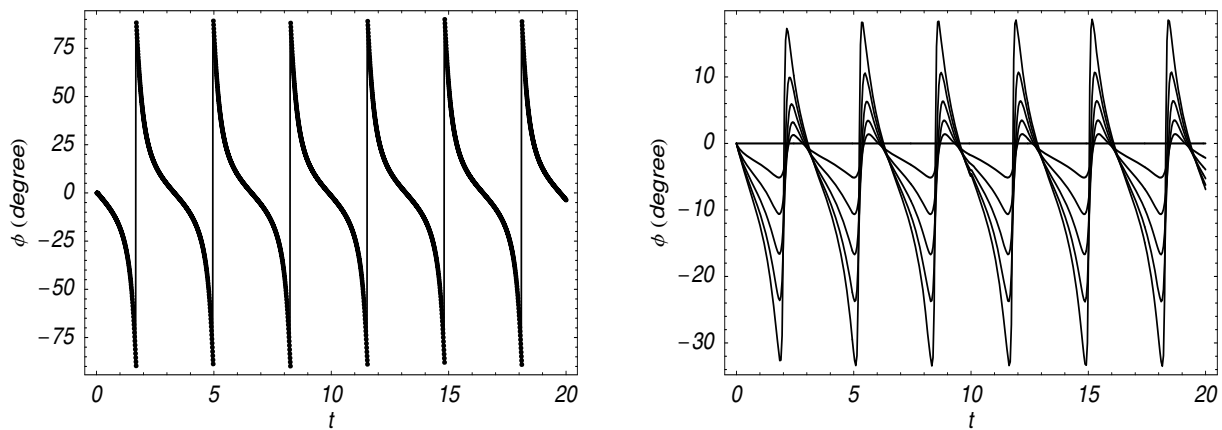

FIG. 10. Left: evolution of the peak orientation angle of LCPs in the middle of the plate gap for the $T W$ attractor $E r=500, D e=4$ with tangential anchoring. Note: $90^{\circ}$ and $-90^{\circ}$ imply the same director orientation. Right: evolution of the peak alignment angle, $\phi(f)(y)$, for six positions $y=1, .98, .96, .94, .92, .90$ near the plates. At $y=1, \phi=0$ due to plate anchoring. The amplitude of oscillation of the peak axis $\phi(f)$ increases with distance from the plates; the extreme curve is $\phi(f)(y=.9)$, at the gap height that is nearly penetrating the defect core, which is associated with strong variations in the shape of the PDF, shown in Figure 11.

tumbling layer. This transition layer has distinguished PDF and stress features and is called a defect, which we now amplify.

To clearly see the defect structure of composite TW periodic states, we show the order parameter $s$ in Figure 11, defined as the difference of the two largest eigenvalues of the second moment tensor $\mathbf{M}, s=d_{1}-d_{2}$. In general, $\mathbf{Q}=\mathbf{M}-\frac{1}{3} \mathbf{I}$ can be written as

$$
\mathbf{Q}=s\left(\mathbf{n}_{1} \mathbf{n}_{1}-\frac{1}{3} \mathbf{I}\right)-\beta\left(\mathbf{e}_{z} \mathbf{e}_{z}-\frac{1}{3} \mathbf{I}\right),
$$

where $\mathbf{n}_{1}=(\cos \phi, \sin \phi, 0)$ is the major director, i.e., the unit eigenvector of $\mathbf{M}$ corresponding to the largest eigenvalue $d_{1} ; \beta$ is the difference of the two smallest eigenvalues, $\beta=d_{2}-d_{3}$; and $\mathbf{e}_{z}$ is the unit vector along the vorticity axis. If $d_{1}=d_{2}$, i.e., $s=0$, then the major director $\mathbf{n}_{1}$ is not uniquely defined, and the principal axes of orientation lie at least in a plane of directions spanned by $\mathbf{n}_{1}$ and $\mathbf{n}_{2}$. Such degenerate PDFs describe a defect state, associated with order parameter degeneracy. At the red spots in Figure 11, the order parameter $s$ reaches 0 , indicating a defect core structure; the wagging layer is between the red spot and the plate. One clearly sees that defect cores are localized pulsating events; they occur as the major director at the outside of the tumbling layer rotates a full cycle, yet the neighboring director in the wagging layer periodically reverses direction. Figure 12 shows the evolution of the order parameter $s$ at several positions in the gap, including the position near the defect center. Results from both parallel and normal anchoring are shown, which shift the gap height of the defect.

Figure 13 shows the normal stress differences $\left(N_{1}\right.$ and $\left.N_{2}\right)$ and shear stress $\left(\tau_{x y}\right)$ associated with defect cores. One finds strong temporal oscillations marked by sign changes across the plate gap! This is a remarkable space-time phenomenon which generalizes the classical experimental observations of Kiss and Porter [18, 19] of sign changes in $N_{1}$ and their subsequent explanation in terms of temporal monodomain steady-unsteady transitions [22]. Here we see clearly that sign changes in stored normal stress differences have a space-time characterization.

We now show two structure transition phenomena induced by Ericksen number 

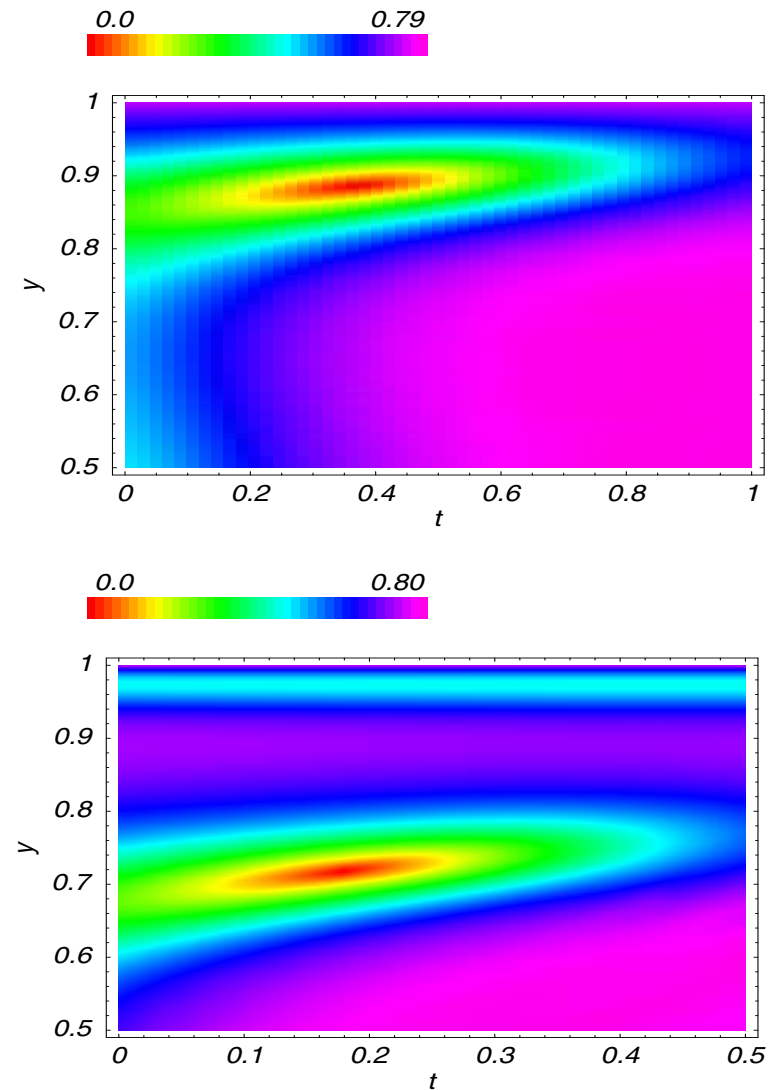

FIG. 11. Order parameter $s$ for the $T W$ attractor for $E r=500, D e=4$. The defect cores are red spots where $s$ drops precipitously toward 0, coinciding with the location where the interior tumbling layer transitions to wagging (finite oscillation of the director) layers that buffer the plates. Top: parallel anchoring. Bottom: normal anchoring.
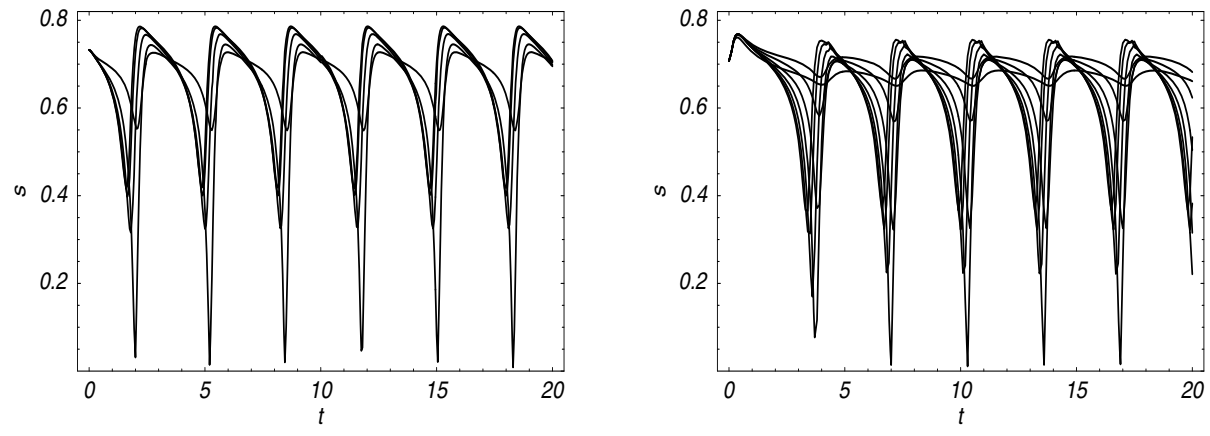

FIG. 12. Evolution of the order parameter s near the defect core of the composite TW attractor for $E r=500, D e=4$. Left: parallel anchoring. Right: normal anchoring.

variations ( $E r$ cascade); the ES-W unsteady structure transition followed by the W-T dynamic structure transition which triggers onset of defect core pulsation. In Figure 14 (left), we plot the height-dependent amplitude of oscillation of the peak PDF 

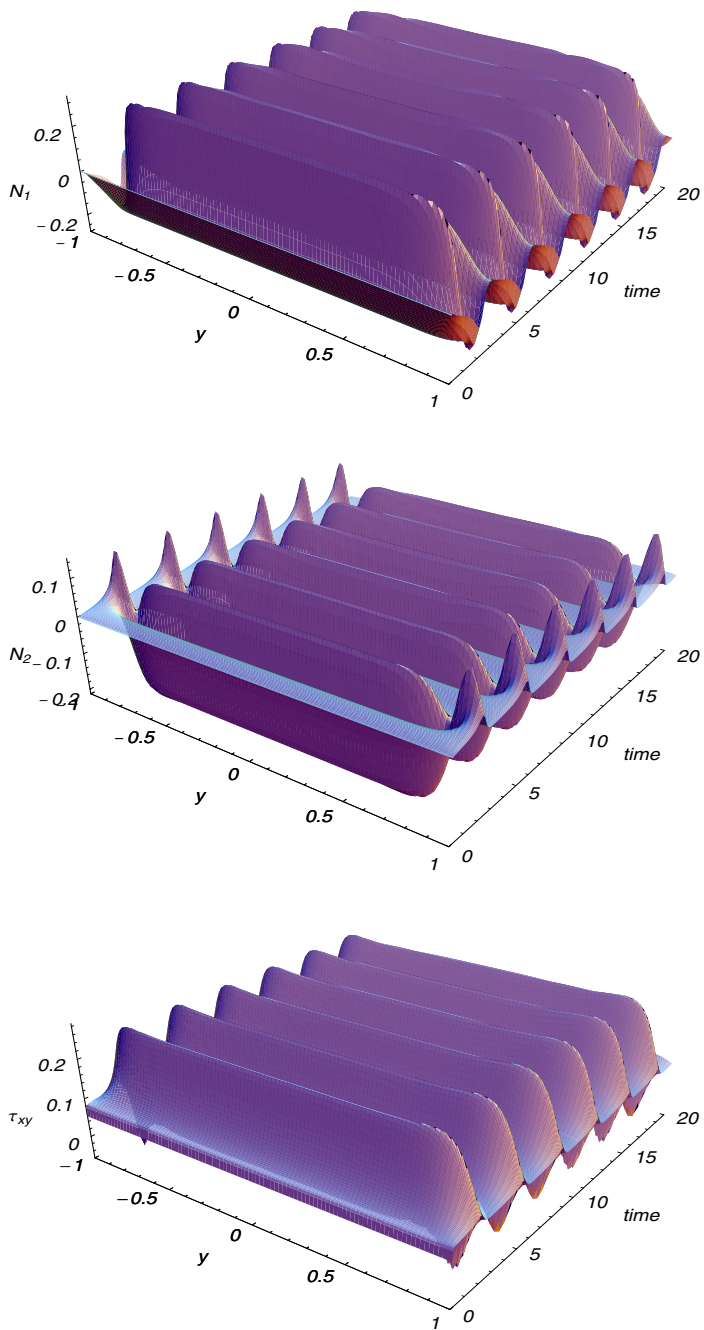

FIG. 13. Gap variations in stored normal stress differences $\left(N_{1}, N_{2}\right)$ and shear stress $\left(\tau_{x y}\right)$ in the time-periodic TW structure attractor for $\mathrm{Er}=500, \mathrm{De}=4$.

alignment angle for $D e=4$ and several $E r$. For $E r=38$, the ES attractor is steady, so at each gap height the attractor oscillation amplitude is zero (not shown in the figure). Beginning at $E r$ around 40, a wagging layer emerges with small oscillation amplitudes. This unsteady attractor is called a wagging (W) structure, by analogy with homogeneous nematic liquids whose PDF oscillates in time under imposed shear. The wagging dynamic structure layer grows in spatial extent, and the major director at the midgap increases in amplitude of oscillation as Er approaches 50. In the range $48<E r<49$, the transition is "stiff," with the amplitude in the middle of the gap growing from below $40^{\circ}$ to the critical transition $90^{\circ}$ (and therefore continuous tumbling), marking onset of the composite TW attractor. This fast structure transition may not be observable in the laboratory; it was not previously reported from secondmoment model simulations (e.g., [31]). After the composite TW state emerges, the 

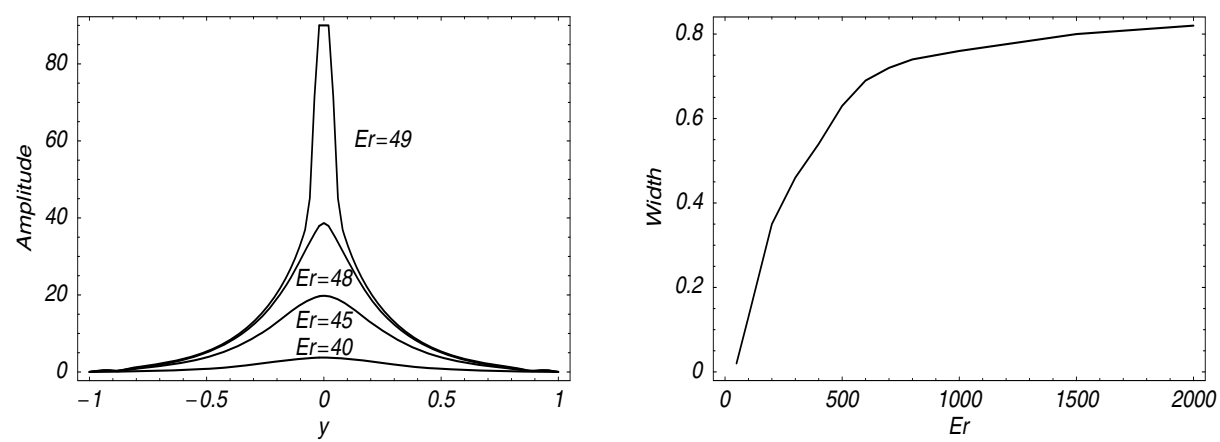

FIG. 14. Left: Ericksen number cascade in the amplitude of oscillation of the major director at each gap height with normal anchoring, for fixed De $=4$, capturing structure transitions from ES attractor $(E r<40)$ to $W$ attractor $(40 \leq E r \leq 48)$, and then to the $T W$ attractor $(E r \geq 49)$. Right: width of the tumbling layer versus $\mathrm{Er} \geq 49$, which also reflects the gap heights of defect core pulsation.

width of the tumbling layer in the center of the gap increases rapidly for parallel anchoring simulations, shown for $E r$ in the range $(50,2000)$ in Figure 14 (right). When $E r=2000$, more than $80 \%$ of the gap is tumbling. As $E r \rightarrow \infty$, only two very thin boundary layers embody the wagging transition between plate alignment and interior tumbling.

Figure 15 shows the structure evolution of the alignment angle (left) and the first normal stress difference (right) during the Ericksen number cascade of Figure 14. The left-hand side features impact conductivity and permeability properties of nematic polymer films $[33,36]$, whereas the right-hand side features impact viscoelastic mechanical properties through residual stress storage.

We turn now to a more detailed study of the $\mathrm{W}$ attractors at $D e=6,8$.

3.4. Wagging periodic states (W structure attractors). We have already seen $\mathrm{W}$ structures as an intermediate state between steady ES and unsteady TW attractors. This transition sequence arises for fixed $D e$ and increasing $E r$, or fixed $E r$ and increasing $D e$, depicted in Table 1. Alternatively, if we fix $E r$ between 50 and 5000 , then increases in $D e$ from $O(1)$ to $O(10)$ lead to the TW to W structure transition, depicted in the columns of Table 1 . We now illustrate this structure transition sequence.

In Figure 16, the top figure (solid line) shows the evolution of the peak PDF alignment angle in the middle of the plates for $E r=500, D e=6$ with parallel anchoring, while the bottom figure shows the peak orientation ("major director") structure evolution across the gap. The dots in the top figure of Figure 16 show the monodomain simulation at the same concentration and shear rate, which coincides with the spatially coupled dynamics. An immediate conclusion is again that the monodomain simulation is an accurate quantitative predictor of the PDF response in the middle of the gap, far removed from the plates, at the start-up from rest states. This property holds as long as elasticity does not overwhelm the monodomain dynamics. This remarkable midgap correlation with homogeneous kinetic dynamics also forebodes the failure of mesoscopic closures, addressed in section 4 , since they all yield nonquantitative accuracy at best and typically miss the correct attractor (see $[8,10,11])$.

Figure 17 shows the structure evolution of the alignment angle (top row) from 

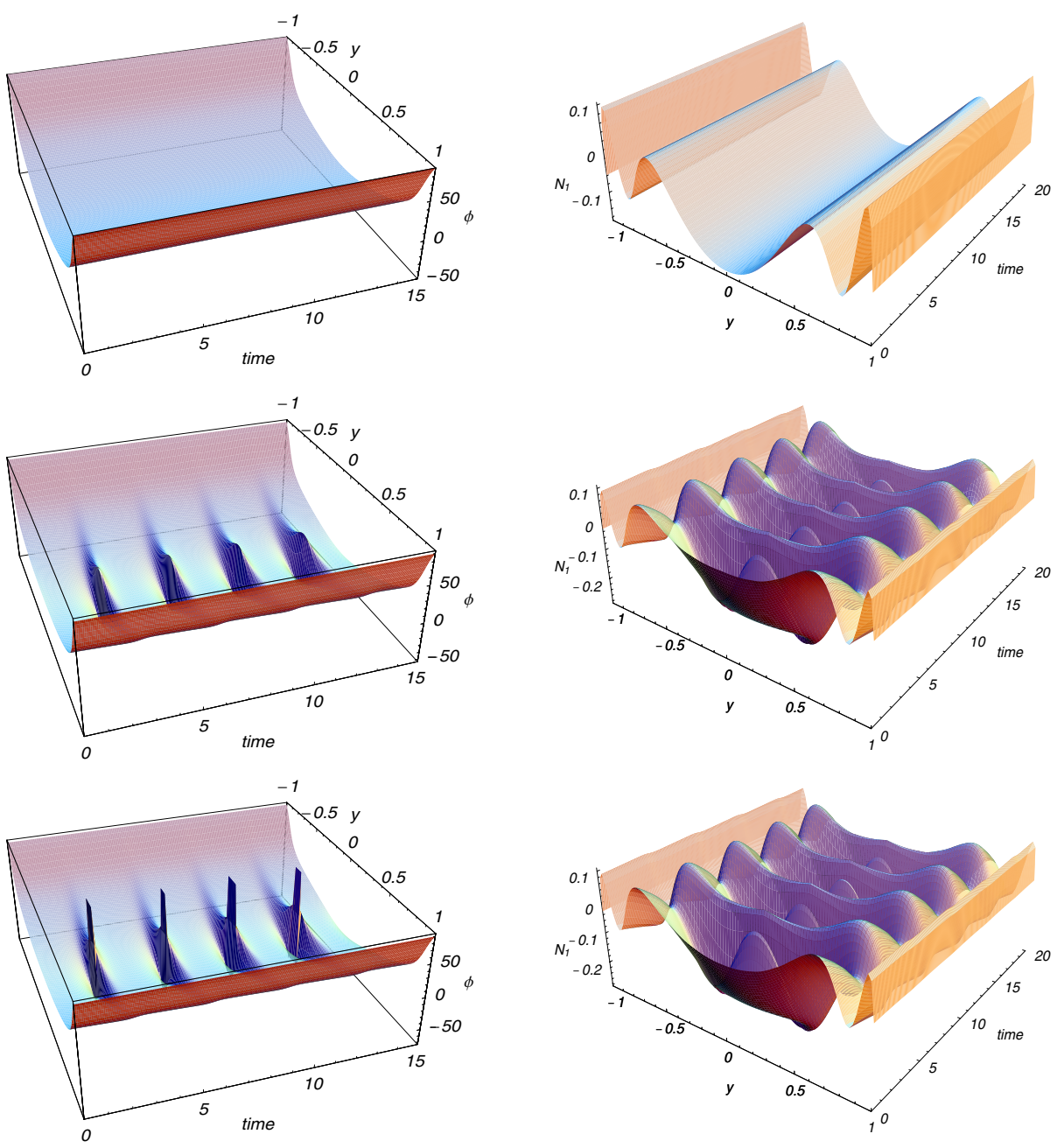

FIG. 15. Time evolution of the alignment angle (left) and the first normal stress difference (right) for the $V S$ at $E r=38, W$ state at $E r=48$, and the composite $T W$ state at $E r=49$. Deborah number $D e=4$ is fixed.

start-up time, along with the degree of alignment (middle row) and the order parameter $s$ (bottom row) for both parallel (left column) and normal anchoring (right column). Compared with TW states, this W structure attractor has much longer wavelength defect structures, with the order parameter $s$ fluctuating between $s=0.82$ and $s=0.04$ uniformly across a large interior layer. This $\mathrm{W}$ attractor therefore has a large interior uniform layer that undergoes strong focusing and defocusing fluctuations typical of wagging monodomains $[35,10,8]$. Again, the midgap behavior is effectively insulated from the boundary layers due to wall confinement, so the strong correlation with homogeneous kinetic predictions raises caution against use of mesoscopic, closure-sensitive approximations.

The normal stress differences and shear stress are shown in Figure 18 for $E r=500$, $D e=6$ with parallel anchoring. The signs of both normal stress differences oscillate in the uniform midgap layers with stress gradients localized near the plates. 

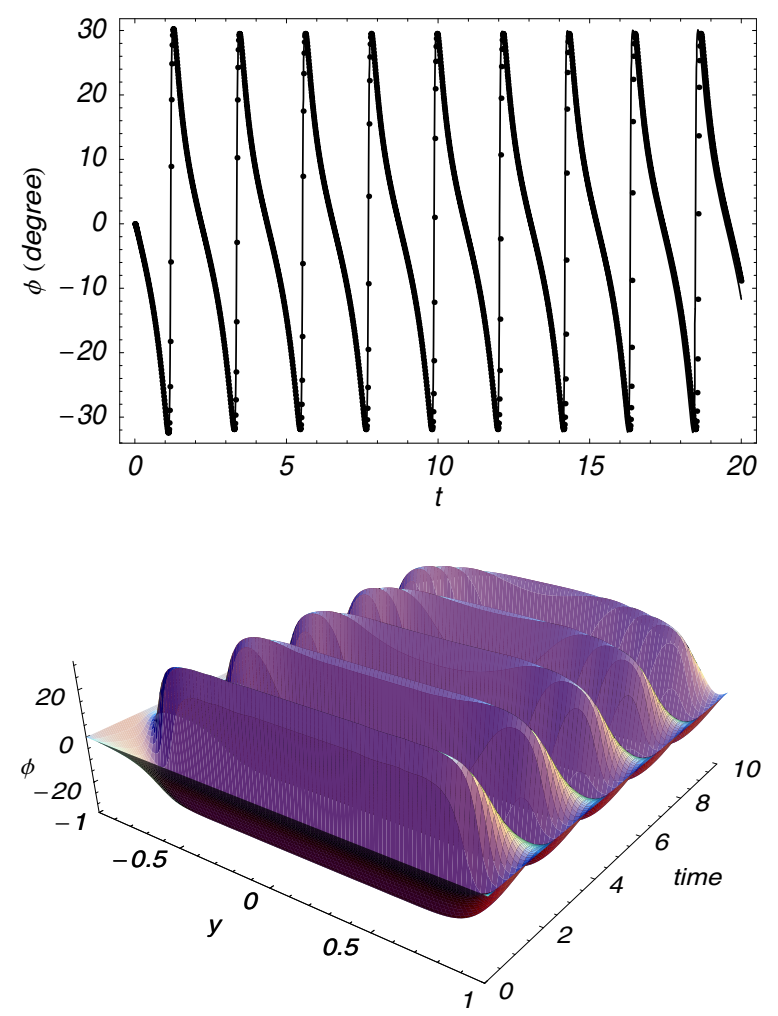

FIG. 16. Top: solid line shows the evolution of the director orientation angle of LCPs in the middle of two plates for $E r=500, D e=6$ with parallel anchoring. Dots show monodomain simulation results with the same De and $\mathrm{Er}=\infty$. Bottom: spatiotemporal structure of the alignment angle.

The steady state transition to unsteady wagging attractors is displayed in Figure 19, which shows the oscillation amplitude of the alignment angle at each gap height for fixed $D e=6$ and increasing $E r$. The steady (ES) attractor at $E r=10$ (not shown) has zero amplitude oscillation across the gap. As $E r$ increases, an interior wagging layer grows in amplitude of oscillation and width, forming a uniform layer across the midgap as $E r \rightarrow 500$. This interior wagging layer slowly consumes the boundary as $E r$ increases toward the infinite $E r$ limit, although $E r \gg 1$ is not numerically resolvable without incredibly expensive spatial resolution and boundary layers on length scales which are not within the resolution of this mean-field theory. From our monodomain simulations, we note that for $E r=\infty$ and $6<D e<8$ the monodomain response is a $\mathrm{W}$ state; therefore the interior of the gap cannot tumble even without spatial coupling. Spatial structure consumes elastic energy, so only $\mathrm{W}$ structures are possible for De between 6 and 8. The difference between Figures $19(D e=6)$ and $14(D e=4)$ is rooted in the monodomain response versus $D e$ which controls the midgap behavior. Again, this feature alone signals caution in using a mesoscopic model that has not been tuned to match kinetic codes in the longwave monodomain limit.

4. Results from mesoscopic model simulations. To compare with mesoscopic model simulations, we use the same parameters as in the kinetic simulation 

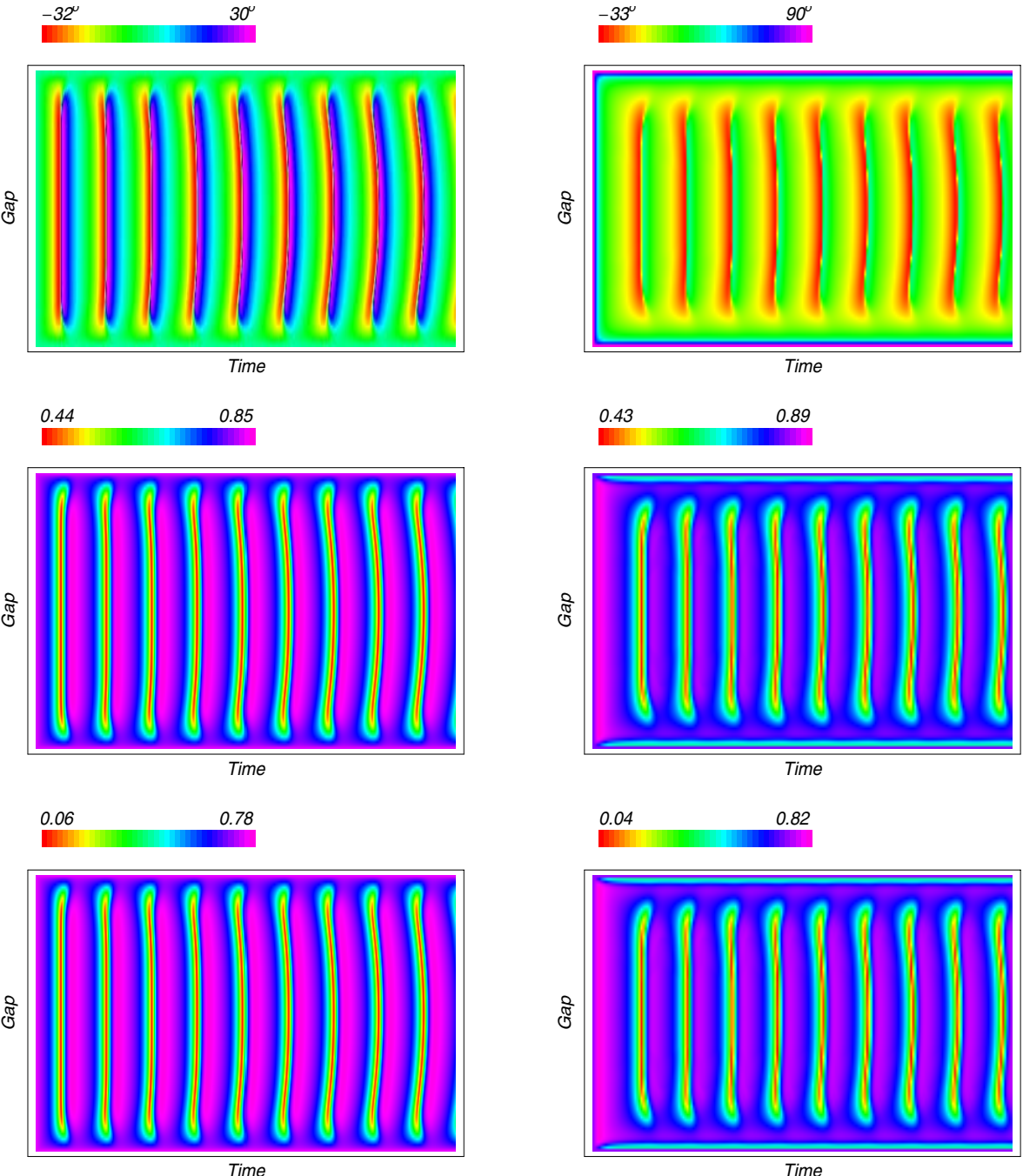

FIG. 17. Contour plots of spatiotemporal structures for $E r=500, D e=6$ from startup to convergence to $W$ attractors. The total time is 20 dimensionless units, or 20 times the mean relaxation time of the nematic liquid. Left column: parallel anchoring. Right column: normal anchoring. Top row: director alignment angle. Middle row: degree of alignment. Bottom row: order parameter $s$.

and consider the Deborah number cascade for fixed $E r=500$ with parallel anchoring. First we use the Doi closure rule, that is, $c_{1}=c_{2}=1$ in the model system (9). In Table 2 we present numerical results for the analogue of the $E r=500$ column of Table 1 versus $D e$. The results are completely different: The unique attractor is ES for $0<D e<6$, which misses the ES-W transition at $D e \sim 0.1$ and misses the W-TW transition at $D e \sim 0.25$. Next, we choose a different closure with $c_{1}=c_{2}=0.6$, which yields a fit to the I-N transition without flow at $N=5$. The results are summarized in Table 3. This mesoscopic model completely misses the steady attractors (ES) at low $D e$, but qualitatively captures the remaining kinetic $D e$ cascade: the transition from $\mathrm{TW}$ to $\mathrm{W}$, and then to VS. 

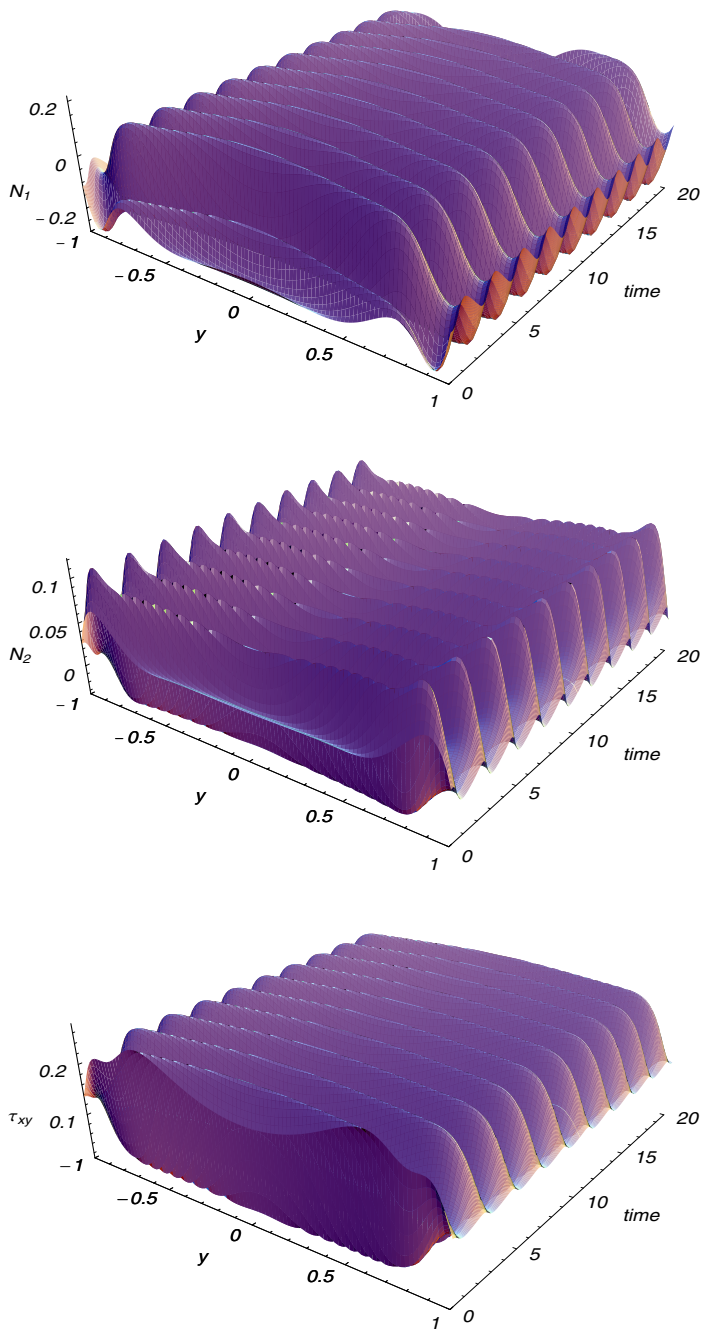

Fig. 18. Normal stress differences $N_{1}, N_{2}$ and shear stress $\tau_{x y}$ for the $W$ structure attractor with $E r=500, D e=6$.

A TW attractor is found at $D e=1$ for the second closure, consistent with the kinetic simulation. The Leslie alignment angle distortion between the plates is shown at several snapshots in Figure 20 (left). In a larger uniform interior layer, the alignment angle oscillates between $-90^{\circ}$ and $90^{\circ}$, showing the continuous rotation of the major director. Near the plates, the angle oscillates at each gap height, with nonuniform structures that fluctuate to interpolate between the end of the tumbling layer and the pinned plate angle. For $D e=4$, the kinetic attractor remains as $\mathrm{TW}$, but the mesoscopic model yields a VS whose final state across the gap is shown in Figure 20 (right). For an intermediate value $D e=2$, the mesoscopic model yields a wagging attractor; the Leslie alignment angle across the gap during a certain time interval is shown in Figure 21. The maximum alignment angle is close to $2^{\circ}$, while the minimum alignment angle is close to $-18^{\circ}$. The median is around $-8^{\circ}$. Note that for the peak 


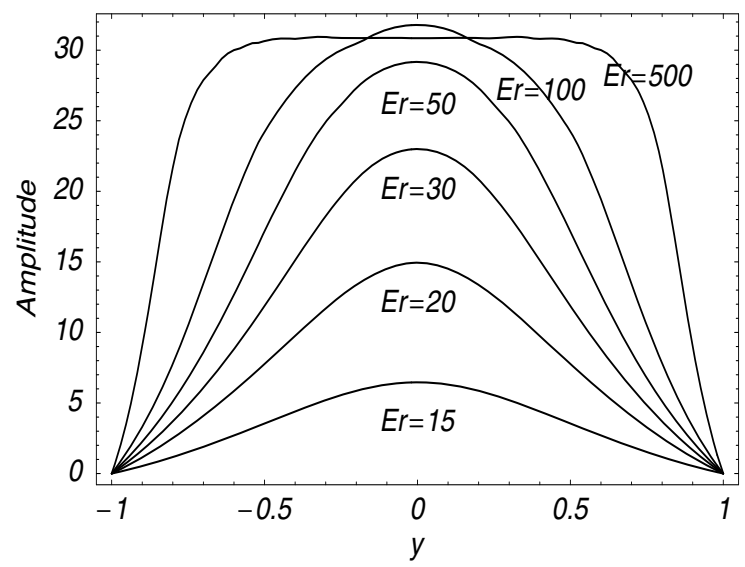

FIG. 19. The Er cascade at fixed De $=6$ from the steady (ES) attractor at Er $=10$ through Er dependent periodic structure $(W)$ attractors for $E r=15$ to 500. Each Er labeled curve depicts the amplitude of major director oscillation at each gap height.

TABLE 2

De cascade structures of the mesoscopic Doi closure model for fixed Ericksen number Er $=500$.

\begin{tabular}{|c||c|c|c|c|c|c|}
\hline \hline$D e$ & 0.01 & 1 & 2 & 4 & 6 & 10 \\
\hline State & ES & ES & ES & ES & ES & ES \\
\hline \hline
\end{tabular}

TABLE 3

De cascade structures of the mesoscopic closure model (9) with $c_{1}=c_{2}=0.6$ for fixed Ericksen number $E r=500$.

\begin{tabular}{|c||c|c|c|c|c|c|}
\hline \hline$D e$ & 0.01 & 1 & 2 & 4 & 6 & 10 \\
\hline State & TW & TW & W & VS & VS & VS \\
\hline \hline
\end{tabular}

alignment angle shown in Figure 16 for the $\mathrm{W}$ state $(D e=6)$ from the kinetic simulation, the median is close to the flow direction. Thus, the peak axis of the PDF is curiously tilted by the closure approximation, even in regimes where the space-time attractor is qualitatively similar to the kinetic attractor.

5. Conclusion. The planar structure attractors of sheared LCPs in parallel plate Couette cells have been characterized in terms of normalized shear rate (Deborah number) and strength of the distortional elasticity potential (Ericksen number). The molecular orientation distribution (PDF), its mesoscopic second-moment features of peak orientation axis and degree of focusing, and stored elastic stresses are combined to characterize the attractors. Four attractor types, two steady and two unsteady, are observed across the parameter domain of $(D e, E r)$. Finally, differences due to parallel versus normal anchoring are illustrated. These structures and their transition sequences in terms of $D e$ (the Deborah number cascade) and $E r$ (the Ericksen number cascade) serve as high-level benchmarks on mesoscopic closure models and as a basis for extensions of these kinetic simulations to include full orientational degrees of freedom, flow feedback, and higher dimensionality in physical space. Phase transitions of structure attractors are identified for both tangential and normal molecular anchoring conditions at the plates. Results presented here serve two purposes for materials 

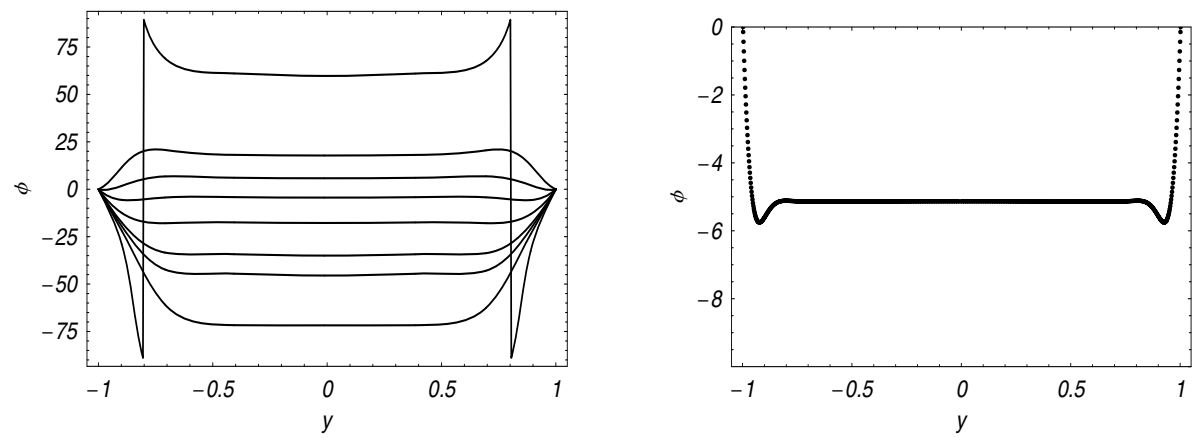

FIG. 20. Simulation results for $E r=500$ from the second closure model ((9) with $c_{1}=c_{2}=$ $0.6)$, which matches the $E r=\infty, D e=0$ isotropic-nematic phase diagram. The left-hand plot shows the Leslie angle for De $=1$ across the plate gap, clearly showing a dynamic, uniform interior tumbling layer consuming $80 \%$ of the shear cell, with wagging nonuniform boundary layers. The right-hand plot shows the steady state director structure for $D e=4$.
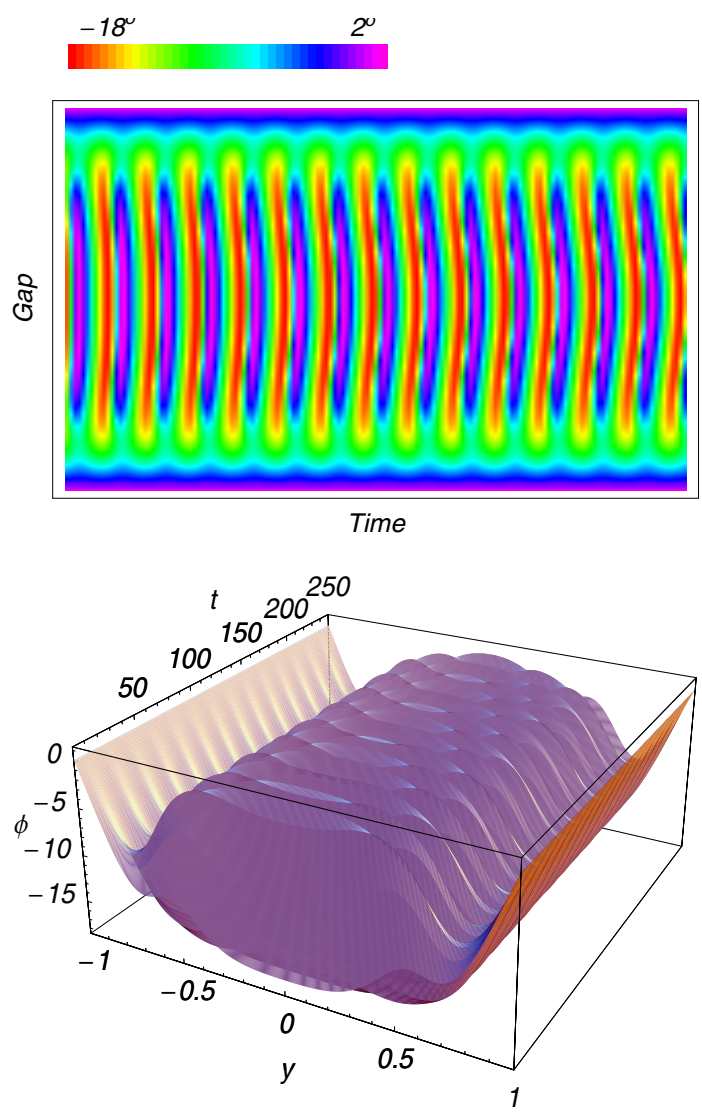

FIG. 21. Simulation results from the closure model equations with De $=2$. The attractor is the wagging state. Top: the color contour plot of the Leslie angle in a time interval. Bottom: the three-dimensional plot of the same quantity. 
engineering. First, the molecular orientational distributions and stored stresses, coupled with homogenization theory of low-volume fraction spheroidal inclusions, give a direct prediction of anisotropic, heterogeneous properties in thin LCP films [33, 36]. Second, the structure attractors and material properties provide a foundation for continuation studies due to release of the controls imposed here. Comparisons with two mesoscopic closure models underscore the extreme sensitivity of kinetic model structure phenomena to closure scheme, a predictable generalization of the monodomain longwave limit $[8,10,11]$.

\section{REFERENCES}

[1] A. N. Beris And B. J. EdwARds, Thermodynamics of Flowing Systems with Internal Microstructure, Oxford Engrg. Sci. Ser. 36, Oxford Sci. Publ., The Clarendon Press, Oxford University Press, New York, 1994.

[2] Z. Cui, M. G. Forest, Q. WAng, And H. Zhou, On weak plane Couette and Poiseuille flows of rigid rod and platelet ensembles, SIAM J. Appl. Math., to appear.

[3] P. G. De Gennes and J. Prost, The Physics of Liquid Crystals, Oxford University Press, Oxford, UK, 1993.

[4] M. DoI, Molecular dynamics and rheological properties of concentrated solutions of rodlike polymers in isotropic and liquid crystalline phases, J. Polym. Sci., Polym. Phys. Ed., 19 (1981), pp. 229-243.

[5] M. Doi And S. F. Edwards, The Theory of Polymer Dynamics, The Clarendon Press, Oxford University Press, London, New York, 1986.

[6] V. Faraoni, M. Grosso, S. Crescitelli, and P. L. Maffettone, The rigid-rod model for nematic polymers: An analysis of the shear flow problem, J. Rheol., 43 (1999), pp. 829-843.

[7] J. Feng, J. TAO, AND L. G. LeAL, Roll cells and disclinations in sheared polymer nematics, J. Fluid Mech., 49 (2001), pp. 179-200.

[8] M. G. Forest AND Q. WANG, Monodomain response of finite-aspect-ratio macromolecules in shear and related linear flows, Rheol. Acta, 42 (2003), pp. 20-46.

[9] M. G. Forest, Q. Wang, H. Zhou, And R. Zhou, Structure scaling properties of confined nematic polymers in plane Couette cells: The weak flow limit, J. Rheol., 48 (2004), pp. 175-192.

[10] M. G. Forest, Q. WAng, And R. Zhou, The weak shear kinetic phase diagram for nematic polymers, Rheol. Acta, 43 (2004), pp. 17-37.

[11] M. G. Forest, Q. Wang, And R. Zhou, The flow-phase diagram of Doi theory for sheared nematic polymers, II: Finite shear rates, Rheol. Acta, 44 (2004), pp. 80-93.

[12] M. G. Forest, Q. Whang, R. Zhou, And E. Chonte, Monodomain response of arbitrary aspect ratio nematic polymers in general linear planar flows, J. Non-Newtonian Fluid Mech., 118 (2004), pp. 17-31.

[13] M. G. Forest, X. Zheng, R. Zhou, Q. Wang, and R. Lipton, Anisotropy and dynamic ranges in effective properties of sheared nematic polymer nano-composites, Adv. Func. Mat., to appear.

[14] M. G. Forest, R. Zhou, And Q. WAng, Full-tensor alignment criteria for sheared nematic polymers, J. Rheol., 47 (2003), pp. 105-127.

[15] M. G. Forest, R. Zhou, AND Q. WANG, Chaotic boundaries of nematic polymers in mixed shear and extensional flows, Phys. Rev. Lett., 93 (2004), 088301.

[16] M. Grosso, R. Keunings, S. Crescitelli, and P. L. Maffettone, Prediction of chaotic dynamics in sheared liquid crystalline polymers, Phys. Rev. Lett., 86 (2001), pp. 31843187.

[17] S. Hess, Fokker-Planck-equation approach to flow alignment in liquid crystals, Z. Naturforsch., 31A (1976), pp. 1034-1037.

[18] G. Kiss And R. S. Porter, Rheology of concentrated solutions of poly ( $\gamma$-benzyl-glutamate), J. Polym. Sci., Polym. Symp., 65 (1978), pp. 193-211.

[19] G. Kiss AND R. S. PorTeR, Rheology of concentrated solutions of helical polypeptides, J. Polym. Sci., Polym. Phys. Ed., 18 (1980), pp. 361-388.

[20] M. Kroger, Simple models for complex nonequilibrium fluids, Phys. Rep., 390 (2004), pp. $453-551$.

[21] R. Kupfermann, M. Kawaguchi, and M. M. Denn, Emergence of structure in a model of liquid crystalline polymers with elastic coupling, J. Non-Newtonian Fluid Mech., 91 (2000), pp. 255-271. 
[22] R. G. Larson, The Structure and Rheology of Complex Fluids, Oxford University Press, New York, 1999.

[23] R. G. Larson and D. W. Mead, The Ericksen number and Deborah number cascades in sheared polymeric nematic, Liquid Cryst., 15 (1993), pp. 151-169.

[24] R. G. LARSON AND H. OtTINGER, The effect of molecular elasticity on out-of-plane orientations in shearing flows of liquid crystalline polymers, Macromolecules, 24 (1991), pp. 6270-6282.

[25] G. Marrucci and F. Greco, Flow behavior of liquid crystalline polymers, Adv. Chem. Phys., 86 (1993), pp. 331-404.

[26] A. D. Rey and M. M. Denn, Dynamical phenomena in liquid-crystalline materials, in Annu. Rev. Fluid Mech. 34, Annual Reviews, Palo Alto, CA, 2002, pp. 233-266.

[27] G. Rienacker and S. Hess, Orientational dynamics of nematic liquid crystals under shear flow, Phys. A, 267 (1999), pp. 294-321.

[28] G. Sgalari, G. L. Leal, And J. J. Feng, The shear flow behavior of LCPs based on a generalized Doi model with distortional elasticity, J. Non-Newtonian Fluid Mech., 102 (2002), pp. 361-382.

[29] Z. TAN AND G. C. Berry, Studies on the texture of nematic solutions of rodlike polymers. 3. Rheo-optical and rheological behavior in shear, J. Rheol., 47 (2003), pp. 73-104.

[30] T. TsujI AND A. D. REY, Effect of long range order on sheared liquid crystalline polymers, Part 1: Compatibility between tumbling behavior and fixed anchoring, J. Non-Newtonian Fluid Mech., 73 (1997), pp. 127-152.

[31] T. Tsuji And A. D. Rey, Effect of long range order on sheared liquid crystalline materials: Flow regimes, transitions, and rheological phase diagrams, Phys. Rev. E (3), 62 (2000), pp. 8141-8151.

[32] Q. WANG, A hydrodynamic theory for solutions of nonhomogeneous nematic liquid crystalline polymers of different configuration, J. Chem. Phys., 116 (2002), pp. 9120-9136.

[33] X. Zheng, M. G. Forest, R. Lipton, R. Zhou, and Q. Wang, Exact scaling laws for electrical conductivity properties of nematic polymer nano-composite monodomains, Adv. Func. Mat., 15 (2005), pp. 627-638.

[34] H. Zhou And M. G. Forest, Anchoring distortions coupled with plane Couette and Poiseuille flows of nematic polymers in viscous solvents: Morphology in molecular orientation, stress and flow, Discrete Contin. Dyn. Syst. Ser. B, to appear.

[35] H. Zhou, M. G. Forest, ANd Q. WANG, Anchoring-induced structure transitions and flow feedback of nematic polymers in plane Couette cells, J. Non-Newtonian Fluid Mech., submitted.

[36] H. Zhou, M. G. Forest, X. Zheng, Q. Wang, and R. Lipton, Extension-enhanced conductivity of liquid crystalline polymer nano-composites, Macromolecular Symposia, to appear.

[37] R. Zhou, M. G. Forest, AND Q. WANG, Kinetic structure simulations of nematic polymers in plane Couette cells. I: The algorithm and benchmarks, Multiscale Model. Simul., 3 (2005), pp. 853-870. 